\title{
Article \\ Coordinated Control of Single-Phase End-Users for Phase Load Balancing in Active Electric Distribution Networks
}

\author{
Gheorghe Grigoraș ${ }^{1,}{ }^{*}$, Livia Noroc ${ }^{1}$, Ecaterina Chelaru ${ }^{1}$, Florina Scarlatache ${ }^{1}$, Bogdan-Constantin Neagu ${ }^{1}$, Ovidiu \\ Ivanov $^{1}$, Mihai Gavrilaș ${ }^{1}$
}

1 Department of Power Engineering; “Gheorghe Asachi” Technical University of Iasi, Romania, ggrigor@tuiasi.ro (G.G.); livia.grimailo@student.tuiasi.ro (L.N.); ecaterina.chelaru@student.tuiasi.ro (E.C.); florina.scarlatache@academic.tuiasi.ro (F.S.); bogdan.neagu@tuiasi.ro (B.C.N.); ovidiuivanov@tuiasi.ro (O.I.); mgavril@tuiasi.ro (M.G.)

* Correspondence: ggrigor@tuiasi.ro;ghgrigoras@yahoo.com Tel.: +04 0232278683 (G.G.)

\begin{abstract}
In the paper, a coordinated control methodology of single-phase (1-P) end-users switching operations on the phases of an active electric distribution network (AEDN) has been proposed to obtain a minimum unbalance degree at the coupling common point (CCP) level with the main distribution system. The phase load balancing (PLB) process considers the smart devices that switch from one phase to another phase the 1-P end-users (consumers and prosumers) to compensate for the phase load unbalance. The proposed methodology has been tested successfully in an AEDN belonging to a Romanian Distribution Network Operator (DNO) containing 114 end-users (104 consumers/10 prosumers) integrated into the Smart Metering System (SMS). The optimal solution leads to a value of the objective function by 1.00004 , represented by the unbalance factor, very close to the ideal target, 1.00. A comparative analysis was conducted considering other possible PLB cases (the consumer-level PLB and prosumer-level PLB), obtaining similar values of the UF (1.027 vs. 1.028), slightly higher than in the hybrid-level PLB. Also, the significant technical benefits were quantified through an energy-saving of $58.73 \%$ and decreasing the phase voltage unbalance rate by $91 \%$ compared to the initial case (without PLB). These results emphasized the positive impact of the proposed coordinated control methodology on the PLB process and evidenced its effectiveness and applicability in the AEDNs.
\end{abstract}

Keywords active electric distribution networks; phase load balancing; coordinated control; smart switching devices; end-users.

\section{Introduction}

In the last period, we are witnessing an accelerated transformation of the electricity distribution sector, and the main component of this irreversible process is the development of electric distribution networks (EDNs). Thus, the classical electric distribution networks must support a radical conversion from the passive to the active area. The volatile character of renewable energy, the high growth of the active consumers (named prosumers), and electric mobility represent the main challenges for the Distribution Network Operators (DNOs) [1].

The active electric distribution networks (AEDNs) mean modernization of the classical EDNs through innovative solutions that lead to network automation and turn it into a system that can integrate with other private and public systems into a connected smart grid [2].

The Clean Energy for All Europeans legislative package [3] asserts that consumers will represent the core of the transition through the AEDNs. More and more consumers will actively participate in the energy market, producing energy for self-consumption and injecting the surpluses into the network. Also, the storage systems can help the con- 
sumers adapting their demand to obtain maximum benefits following the prices or other incentives.

Digital networks, the Internet of Things, electric mobility, distributed generation, and energy efficiency are challenges that involve significant changes in the AEDNs. All these challenges identify with the digital "revolution" that involves two-way communication that allows analysis and transmission of high data amounts and coordination in real-time all devices integrated into the network. The digitalization process represents the future of AEDNs, which must be flexible, efficient, and reliable to ensure the continuity of the energy supply at the end-users [4].

In this context, the planning and operation activities have changed, being increasingly complex. The AEDNs must efficiently integrate all types and categories of the energy sources and end-users, covering all their actions and requests. Among the characteristics of the AEDNs can be highlighted the following: integrating the all generation sources and storage systems; including the smart metering system (SMS); ensuring the conditions for the bidirectional energy flows; the flexibility to changes in the structure of the topology and end-users; ensuring the power quality; securing the data communication systems [5], [6].

Through the optimal operation and planning activities carried out by the DNOs, the AEDNs have lower power losses, allow remote operations when failures occur (detecting being more quickly) and fast restoration of electricity supply, and, also, can provide the absorption of energy from prosumers. Electric vehicles can be charged from the grid and could inject energy into the grid when the sale of electricity would be profitable. The smart meters installed at the end-users allow real-time communications capable of "selfrepair" and ensure rapid recovery from power outages, facilitate market operations and provide the consumer with the opportunity to choose and be informed.

As presented, the AEDNs integrate a high number of 1-P consumers, with a dynamic variation of the demand, and 1-P prosumers with different generation technologies, connected to the three phases, so that the phase currents are no longer equal, leading to an unbalance, see Figure 1.

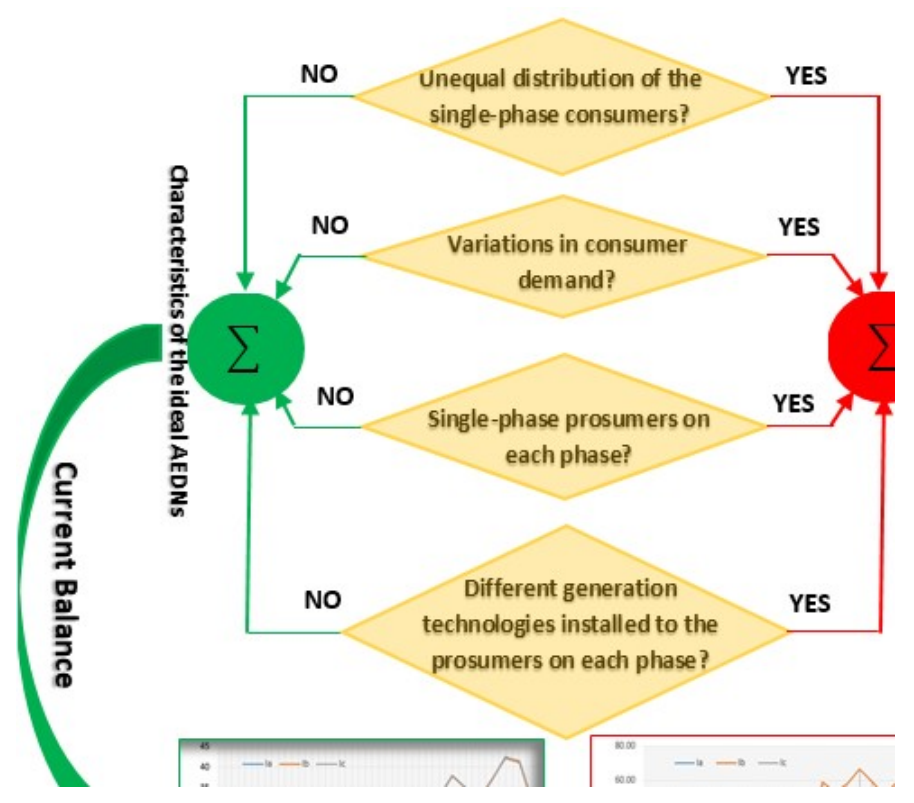

Figure 1. The causes of the current unbalance in the AEDNs

Figure 2 presents the effects of the current unbalance represented by exceeding the admissible limits of the phase-neutral and phase-phase voltages, the difficulties in the 
voltage control (through the taps of the MV/LV transformer), and the possibility to propagate the unbalance in the MV network of the DNO can be identified [7], [8].

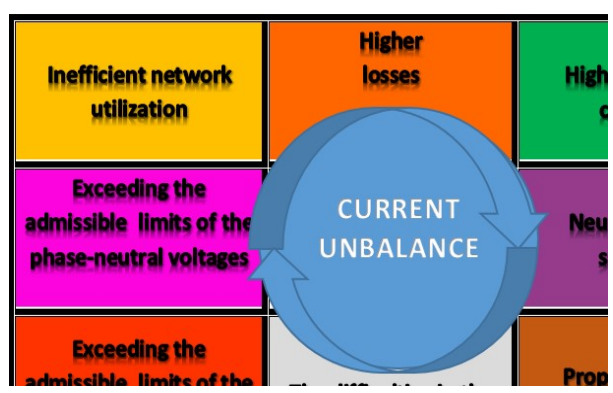

Figure 2. The effects of the current unbalance

A strong linkage between the current and voltage unbalances can be emphasized, with many situations in which one type of unbalance leads to the other.

To attenuate these effects and to operate optimal the AEDNs, the DNOs apply measures that involve load compensation, network reconfiguration, and phase swapping of the 1-P end-users. The first two measures have excellent results in the MV networks [9] - [12]. The third, associated with a phase load balancing (PLB), can lead to significant technical and economic benefits in the LV AEDNs, especially if the single-phase end-users (consumers and prosumers) have installed Smart Switching Devices (SSDs) to swap the connection phase such that to minimize the current unbalance [13] - [15].

The paper concentrates on the phase swapping measure, proposing an original centralized control methodology of the SSDs installed to all 1-P end-users to provide a fast PLB process in the AEDNs. The DNOs can have the control capability of SSDs due to the advanced technologies, which today increased at a high level through a communication interface (CI) between end-user and network. The CIs communicate with a control module (CM) from the common coupling point (CCP) with the distribution system (usually, the electric distribution substation, EDS), which manages the 1-P end-users by proper instructions and controls the SSDs, see Figure. 3.

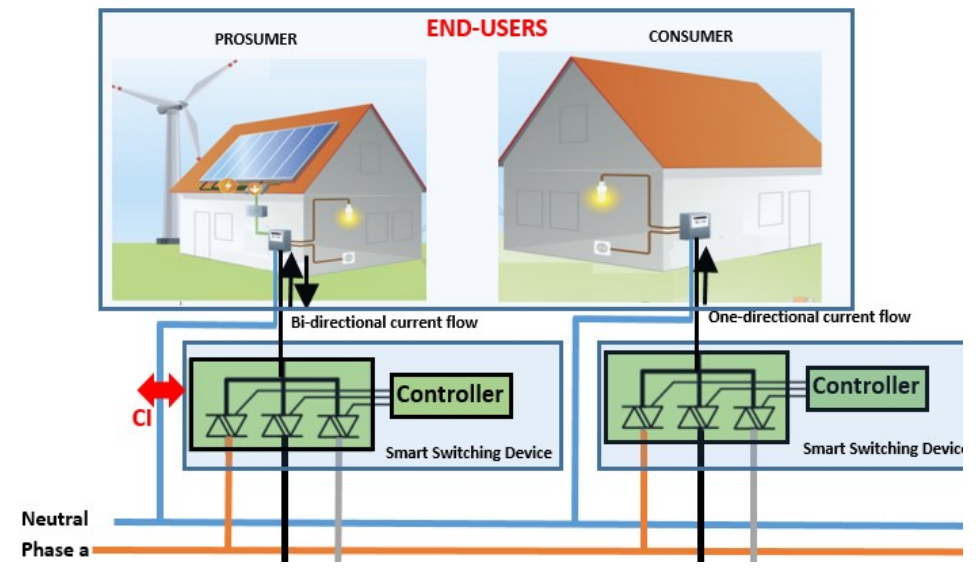

Figure 3. The centralized control of the Smart Switching Devices 


\subsection{Related Literature}

In the last years, the increase of the small-scale local renewable generation sources has led to a new electricity supply infrastructure. According to this innovative concept, the electricity generation is done through small-size power units located near consumers or even to consumers (prosumers) and connected to the network, witnessing a transition from traditional passive networks to AEDNs. The PLB is a significant technical problem that must be solved by the DNOs, representing, also, a necessity to operate the AEDNs optimally. Most of the approaches presented in the literature in the last years use classical consumer-level balancing. The representative solutions based on the phase-swapping devices are proposed in [16] - [20]. Also, the approaches presented in [21] and [22] formulate the PLB problem as a multi-objective optimization problem that minimizes the neutral current at the desired points of the circuit, the energy losses, and the number of reconnected elements. Singh et al. used in [23] the dependency voltage-load to solve the PLB problem in a classical radial distribution network considering Particle Swarm Optimization. The combinatorial optimization, together with fuzzy logic and NewtonRaphson, has been used in [24] by Siti et al. to identify the solution associated with the PLB problem. A control methodology based on a load-electricity transfer index has been proposed [25], including an analysis of three-phase unbalanced control measures.

Of course, there are many others references, but they differ only through the used PLB algorithm that could lead to the real-time PLB solutions. With the occurrence of prosumers, the solutions considered their integration in the PLB process. A control strategy of three-phase prosumers which integrates the interfaces provided by the gridinteractive inverters to control the current injections on each phase represents the base of the solution proposed in [26] to compensate for the current unbalance. Reference [27] presented a similar concept applied to single-phase prosumers. Because the strategy did not consider the locations of the prosumers in the AEDN, the probability of some imbalances in certain areas could be high. Blaud et al. performed in [28] a study based on a modelling of the multi-energy systems and an advanced economic model predictive control to identify the optimal solution of the PLB problem. Another study examined the capability of a multi-prosumer node containing the small-size generation units, loads, storage from two-energy carriers to participate in a PLB process. The analysis performed in [29] by Mieński et al. considering a 3-P prosumer had as purpose determination of the optimal operating profiles associated with a generation system containing an energy storage unit to participate in the PLB process. The issue of dynamic phase balancing in an AEDN has been treated in [30], proposing the modified shuffled frog leaping algorithm to minimize the power loss and improve the current unbalance.

The research from [31] pointed out the characteristics of the LV AEDNs regarding the technical requirements for connection and the power quality associated with the integration of the prosumers without analyzing the PLB problem. A solution based on the controllers for the PV generation systems has been proposed in [32] and tested in a distribution system with looped feeders.

The approach proposed in [33] by Charalambous et al. solved two problems simultaneously regarding the power factor compensation and the current unbalances using advanced functionalities enabled by grid-tied inverters of photovoltaics and energy storage systems. An electronic device controls both reactive power and active power flow on the phases according to the measured loading conditions in the CCP. Ciontea and Iov investigated in [34] the influence of load data on power quality studies focusing on load imbalance level and voltage quality in a low voltage EDN. Kwaśny and Zieliński proposed in [35] a power electronic AC/DC converter together with a multi resonant control algorithm for prosumer applications in a four-wire topology with a DC bus, which couple the converter to a renewable energy source and energy storage. The solution proposed in [36] considered a system for dynamic control of the RMS value and phase angle of the voltage source against the phase voltage of the LV grid, simultaneous with the current phase angle issued by the power source against voltage. Volosciuc and Dragosin 
proposed in [37] an algorithm that coordinates the operation of data centres and distributed energy resources to reduce current unbalance and improve reliability and efficiency. The authors used a modified IEEE distribution test feeder to test the proposed algorithm.

Table 1 presents a summary of the references with an emphasis on the implementation level of the solution: consumer/prosumers, testing network type (EDN/AEDN), balancing procedure (manual/remote control), implementation mode (real-time/offline), and objective proposed.

Table 1. A comparative state-of-the-art of the proposed PLB approaches

\begin{tabular}{|c|c|c|c|c|c|}
\hline \multirow[t]{2}{*}{ References } & \multicolumn{2}{|c|}{$\begin{array}{c}\text { Implementation level of } \\
\text { the PLB solution }\end{array}$} & \multirow[t]{2}{*}{ Testing } & \multicolumn{2}{|c|}{$\begin{array}{l}\text { Implementation } \\
\text { possibility }\end{array}$} \\
\hline & Consumer & Prosumers & & Real-Time & Offline \\
\hline $\begin{array}{l}{[16],[19],[22],[23],} \\
{[24],[25],[34]}\end{array}$ & $X$ & - & Test EDN & - & $X$ \\
\hline$[17]$ & $X$ & - & Laboratory configuration & - & $X$ \\
\hline [18], [21] & $X$ & - & Laboratory configuration & $X$ & - \\
\hline [20] & $X$ & - & Real EDN & $X$ & $x$ \\
\hline [30] & $x$ & - & Real EDN & $x$ & - \\
\hline [26], [27] & - & $X$ & Real AEDN & $X$ & $X$ \\
\hline [29], [33] & - & $X$ & Laboratory configuration & $X$ & - \\
\hline [31], [32] & - & $X$ & Real AEDN & - & $X$ \\
\hline$[35],[36]$ & - & $X$ & Simulation & - & $X$ \\
\hline
\end{tabular}

\subsection{Our Contributions}

Although many studies analysed the PLB problem in the classic EDNs or AEDNs, the perspectives developing a general methodology applied regardless of the end-user type (consumer-level, prosumer-level, or mixed) have not yet been exploited. In this context, the authors proposed a new methodology based on the centralized control of the SSDs to identify PLB solutions regardless of the type, structure, and participation degree (through the availability or unavailability of the SSDs) of the end-users (consumer or prosumer). From the literature review, the methodology would represent the first approach that ensures the flexibility of the PLB process by the possible combinations between absorbed and injected currents by the consumers and prosumers in the three phases. The main contributions of the proposed methodology refer to the following:

- Implementing a procedure to real-time work for the simultaneous query of three different databases (Topology Database, SMS Database, and End-Users Database) of the DNO for uploading the necessary input data in the PLB process. This procedure allow fast data processing.

- Proposing an original PLB algorithm applied for other possible PLB cases encountered in the AEDNs: 1-P consumer-level (C-PLB), 1-P prosumer-level (P-PLB), or hybrid-level (H-PLB) for all 1-P end-users (prosumers and consumers), running regardless of the status of the SSDs (available or unavailable) and the participation degree of the end-users (depending on the absorbed/injected current, equal or different to 0 , the 1-P end-user is or not considered in the PLB process).

- Conducting a complex study where all possible PLB cases represented by C-PLB, P-PLB, and H-PLB have been compared to confirm the accuracy of the proposed methodology regardless of the structure and participation degree of the end-users.

The methodology can produce meaningful benefits in the AEDNs that integrate a high-speed data communication infrastructure and a master controller in the CCP, having the functions associated with high data acquisition and processing speed.

\subsection{Paper organization}


The structure of the paper includes the following sections: Section 2 introduces details regarding each stage from the framework of the proposed methodology; Section 3 presents a complex study performed in a real AEDN from Romania to highlight the accuracy of the methodology in the three possible scenarios (cases) represented by 1-P consumer-level PLB (C-PLB), 1-P prosumer-level PLB (P-PLB), and the hybrid-level (HPLB); and Section 4 contains the conclusions, emphasizing, also, the future works.

\section{Methodology}

The proposed methodology allows the coordinated control of the switching devices installed to all 1-P end-users connected to three-phase four-wire AEDN to perform a fast PLB process. It always leads to the best PLB solutions considering the availability or temporary unavailability of the SSDs and the participation degree of the end-users, according to the value of absorbed/injected current (equal or different to 0). Figure 4 presents the flowchart associated with the proposed methodology.

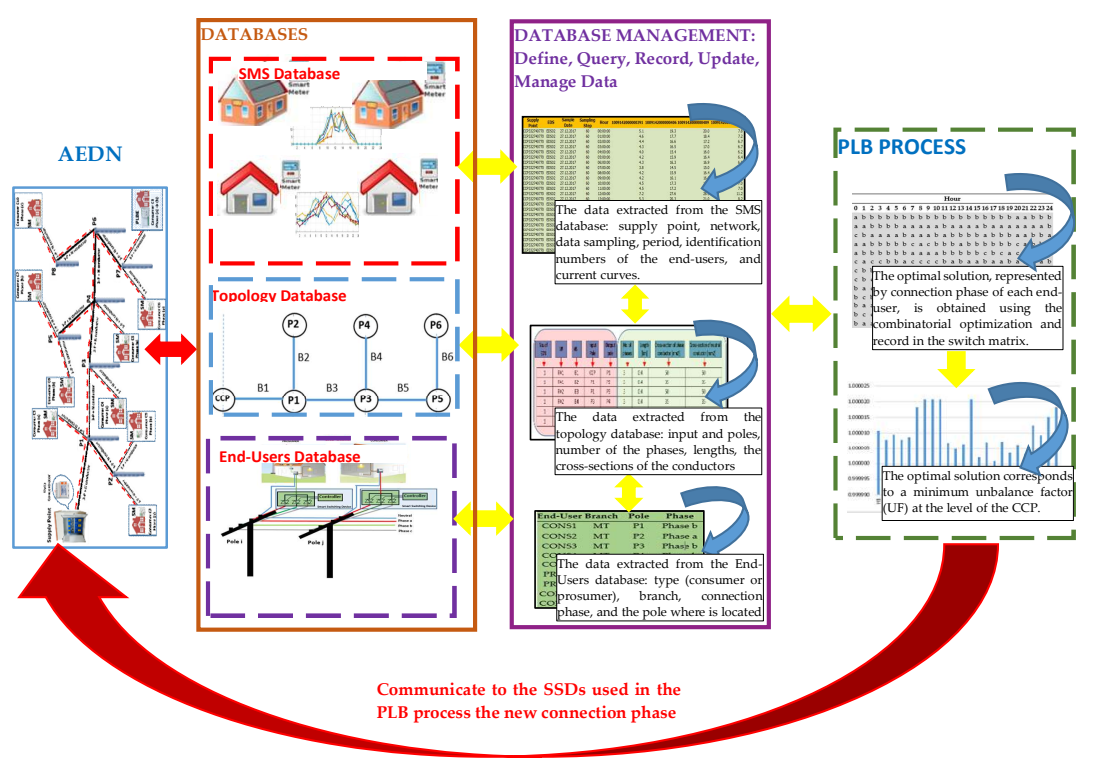

Figure 4. The flow-chart of the proposed coordinated control methodology

Uploading all required information in the PLB process is done from three databases available at the level of the DNO (Topology Database, SMS Database, and End-Users Database). These refer to the topology, identified through the technical and location characteristics of the main elements (poles and conductors) associated with the analysed AEDN, the current profiles of the end-users provided by smart meters identified through the identification numbers, and the end-users regarding the type (consumer or prosumer), the connection phase, and the pole where is located.

The PLB process is based only on the phase swapping of the 1-P consumers and prosumers. For each pole, the optimal local solution, represented by the connection phase of each end-user (consumer/prosumer), is identified with the help of combinatorial optimization. The objective is to minimize the hourly unbalance factor, starting with the end poles and stopping with the EDS, subject to the constraints referring to the phase currents and the number of the end-users connected. Finally, the communication module transmits the new connection phases to the SSDs used in the PLB process. The following paragraphs detail each stage.

\subsection{Database Management}




\subsubsection{Topology Database}

Let a radial (tree) AEDN, with $N l$ poles, $n b$ branches, and a single bus-slack (representing the common coupling point (CCP) with the network operated by the DNO). Under these conditions, $n b-N l+1=0$, and accordingly, $n b=N l-1$.

The topology of the AEDN must be recognized quickly, such that the PLB process takes place in real-time. The algorithm proposed in [38] has been used inside the methodology due to satisfying this feature. Two vectors participate in recognizing the topology: the first vector, $[V P]$, having as elements the number of sections from each partition of the AEDN, and a second vector, $[V B]$, containing the name of sections belonging to each partition placed in ascending order. As an example, the topology sequence of the proposed methodology containing details on the recognition process associated with an AEDN with seven poles, including the one on which the EDS is mounted (representing the $(C P)$, and six sections, see Figure 5, based on the vectors $[V P]$ and $[V B]$, to which is added the information from the DNO 's database regarding the length, number of the phases, the cross-section of the conductors (phase and neutral). Figure 6 contains the topology sequence attached to the AEDN with seven poles.

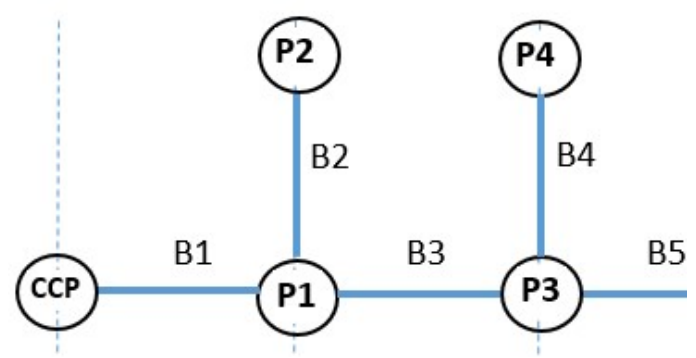

Figure 5. An AEDN with seven poles (including $\mathrm{CCP}$ ) and six sections

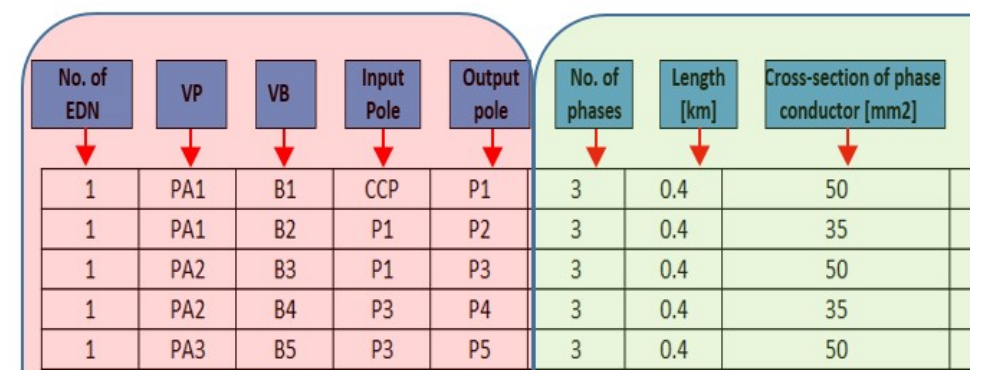

Figure 6. The data sequence extracted from the topology database

The elements of the vector $[V P]$ are associated with the partitions $P A 1, P A 2$, and $P A 3$. Based on these, the vector $[V B]$ is built, containing the elements $B 1$ and $B 2$ (assigned to partition PA1), $B 3$ and $B 4$ (assigned to partition $P A 2$ ), and $B 5$ and $B 6$ (assigned to partition $P A 3)$. Also, the End-Users database is accessed and for each AEDN.

The data uploaded from the topology database are stored in the following input vectors used by the PLB algorithm:

$[\mathrm{Li}]$ - the input pole of each branch, size $(n b \times 1)$,

$[L o]$ - the output pole of each branch, size (nbx1),

$[N p]$ - the number of the phases between each two poles, size $(n b \times 1)$,

$[L]$ - the length between each two poles, size $(n b \times 1)$,

$[C S p]$ - the cross-section of the phase conductors between each two poles, size $(n b \times 1)$,

$[C S n]$ - the cross-section of the neutral conductor between each two poles, size $(n b \times 1)$. 


\subsubsection{SMS and End-Users Database}

The second stage targets establishing the linkage with the database of the DNO attached to the SMS that contains the information regarding the values of the absorbed/injected currents by the consumers and prosumers. Figure 7 presents, as an example, a data sequence uploaded from the SMS database containing the following fields: Supply Point (associated with the identification number of the smart meter installed in the EDS), EDS (the name of the electric distribution substation which supplies the AEDN), Sample Data (the period queried by the Decision Maker), Sampling Step (15, 30, or 60 minutes), Hour (the time of data recording), "1009142000000391" (the identification number of the smart meter installed at an end-user).

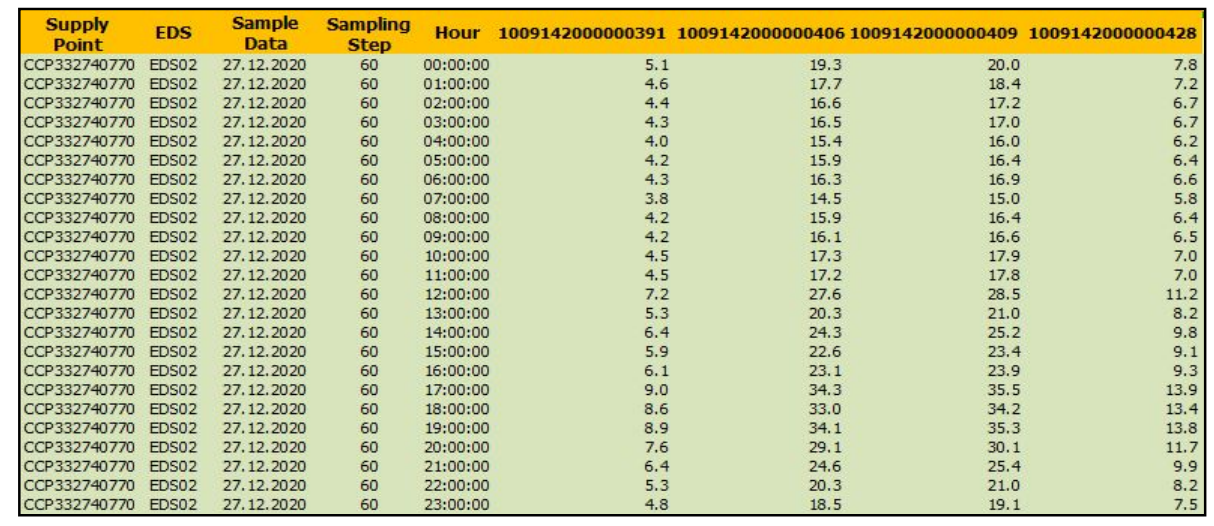

Figure 7. The data sequence extracted from the database of the SMS

The signification of each record is described below:

- Supply Point is associated with each EDS through the identification number of the data concentrator having as principal function to collect the data read from the smart meters installed at the end-users allocated to the EDS. This point represents the link with the electric distribution network operated at the medium voltage by the DNO.

- EDS: This record allows the Decision Maker to locate the EDS in the network based on its name. Also, the information offers the opportunity to identify extremely fast the supplied HV/MV electric substation.

- Sampling Step: It has a significant influence on data processing. The sampling step of the load curve can be programmable between 5 and 60 minutes (the normative values being 5, 15, 30, 60 minutes). The data sampling of the smart meters installed by the DNOs to the end-users is 60 minutes in most cases.

- Hour: This field contains the information corresponding to the time with individual hour, minute, and second components (hh:mm:ss) of the recording the value of the load (current or power).

- The next columns are assigned to the loads (current or power) recorded and transmitted by each smart meter recognized through an identification number in the database (for example, 1009142000000391).

The query considers the filters associated with the Supply Point and Sample Data fields and, depending on Sampling Step (in this case, 60 minutes), accesses the values of the currents from the smart meters, identified through the identification numbers attached to the analyzed AEDN, sending them to the data processing module..

Also, the information attached to each end-user regarding the type (consumer or prosumer), the connection phase, and the pole is uploaded from the End-Users database using the identification number of the smart meter assigned in the SMS database.

Recording the information for the PLB process is done in the following vector data structures: 
[ID] - the identification number of the smart meter associated with the end-users, size (Nux1),

$[S U]$ - the status of the PLB devices (value $0(O f f)$ and value $1(O n))$, size $(N u x 1)$,

$[C C]$-the hourly phase currents of the end-users, size (Nux1),

$[T U]$ - the type of the end-users (1 - consumers and 2 - prosumers), size (Nux1),

$[p]$ - the connection phases of the end-users $(a, b$, and $c$, for the 1-P end-users, and $a b c$, for the 3-P end-users), size ( $N u \times 1)$,

$[N l]$ - the poles where are assigned the end-users, size $(N u \times 1)$,

where $n b$ is the number of the branches from the AEDN and $N u$ signifies the number of the end-users, including both consumers and prosumers.

\subsection{The PLB mathematical model}

The proposed PLB algorithm can be used only for the phase swapping of the 1-P consumers and prosumers connected to a radial AEDN. It aims to minimize the hourly unbalance factor (UF) at the level of each pole, starting with the end poles and stopping with the the EDS, subject to the constraints referring to the phase currents and the number of the end-users connected. The necessary data used in the PLB process are extracted from the input vectors, and their significance inside the algorithm is the following:

$T$, the analysed time interval (in our case study, $T=24$ hours);

$p$, the connection phase of the end-users to the AEDN, $p \in\{a, b, c\}$;

$n_{p}$ - the number of the phases (in the AEDNs with four-wires, $n_{p}=3$ );

$N_{l}$, the number of the poles from the AEDN, including, also, the pole where is mounted the electric distribution substation (EDS);

$N_{c, l}$ the number of the consumers connected to the pole $l, l \in\left\{1, \ldots, N_{l}\right\}$;

$N_{P, l}$, the number of the prosumers connected to pole $l, l \in\left\{1, \ldots, N_{l}\right\}$;

$N u, l$, the total number of the end-users (prosumers and consumers) connected to pole $l, l$ $\in\left\{1, \ldots, N_{l}\right\}$;

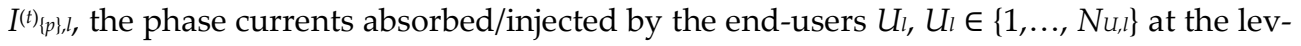
el of the pole $l, l \in\left\{1, \ldots, N_{l}\right\}$, at the hour $t, t \in\{1, \ldots, T\}$;

$I^{(t)}\{p\}, C, l$, the phase currents absorbed by the consumers $C_{l}, C \in\left\{1, \ldots, N_{C, l}\right\}$, connected to the pole $l, l \in\left\{1, \ldots, N_{l}\right\}$, at the hour $t, t \in\{1, \ldots, T\}$;

$I^{(t)}\{p\}, P, l$, the phase currents injected/absorbed by the prosumers $P_{l}, P_{l} \in\{1, \ldots, N P, l\}$, connected to pole $l, l \in\left\{1, \ldots, N_{l}\right\}$, at the hour $t, t=1, \ldots, T$;

$I^{(t)}\{p\}, P,(l+1)$, the phase currents aggregated at the pole $(l+1)$ downstream by the pole $l, l \in\{1$, $\left.\ldots, N_{l}\right\}$, at the hour $t, t=1, \ldots, T$ (for the end poles the value is 0 ).

A variable $\alpha^{(t)}\{p\}, l$, having the value 1 or 0 , has been defined to determine if the 1-P end-user (prosumer or consumer) is or not connected on the phase $p$, at the hour $t$.

The objective function has the expression,

$$
\min \left(U F_{l}^{(t)}\right)=\min \left(\frac{\sum_{p \in\{a, b, c\}}\left(\frac{I_{\{p\}, l}^{(t)}}{I_{m, l}^{(t)}}\right)^{2}}{n_{p}}\right)(\forall) t \in\{1, \ldots, T\},(\forall) l \in\left\{1, \ldots, N_{l}\right\}
$$

where:

$$
I_{m, l}^{(t)}=\frac{\sum_{p \in\{a, b, c\}} I_{\{p\}, l}^{(t)}}{n_{p}}(\forall) t \in\{1, \ldots, T\},(\forall) l \in\left\{1, \ldots, N_{l}\right\}
$$

and the constraints referring to the phase currents and the number of the end-users connected are the following:

$$
I_{\{p\}, l}^{(t)}=I_{\{p\}, C, l}^{(t)}+I_{\{p\}, P, l}^{(t)}+I_{\{p\},(l-1)}^{(t)},(\forall) t \in\{1, \ldots, T\},(\forall) l \in\left\{1, \ldots, N_{l}\right\}, p \in\{a, b, c\}
$$




$$
\begin{aligned}
& I_{\{p\}, C, l}^{(t)}=\sum_{i=1}^{N_{\{p, C, l}^{(t)}} \alpha_{\{p\}, l, i}^{(t)} \cdot I_{C, l, i}^{(t)},(\forall) t \in\{1, \ldots, T\},(\forall) l \in\left\{1, \ldots, N_{l}\right\}, p \in\{a, b, c\} \\
& I_{\{p\}, P, l}^{(t)}=\sum_{j=1}^{N_{p p, P, l}^{(t)}} \alpha_{\{p\}, l, j}^{(t)} \cdot I_{P, l, j}^{(t)},(\forall) t \in\{1, \ldots, T\},(\forall) l \in\left\{1, \ldots, N_{l}\right\}, p \in\{a, b, c\} \\
& N_{C, l}=\sum_{p \in\{a, b, c\}} N_{\{p\}, C, l}^{(t)},(\forall) t \in\{1, \ldots, T\},(\forall) l \in\left\{1, \ldots, N_{l}\right\} \\
& N_{P, l}=\sum_{p \in\{a, b, c\}} N_{\{p\}, P, l}^{(t)},(\forall) t \in\{1, \ldots, T\},(\forall) l \in\left\{1, \ldots, N_{l}\right\} \\
& N_{U, l}=N_{C, l}+N_{P, l},(\forall) l \in\left\{1, \ldots, N_{l}\right\}
\end{aligned}
$$

For a pole $l, l=\left\{1, \ldots, N_{l}\right\}$, the optimal local solution $p^{*}$, represented by connection phase of each end-user (consumer/prosumer), is obtained using the combinatorial optimization [39].

Formally, a combinatorial optimization problem associated with the PLB process refers to a set of possibilities to which the objective given by the relation (1) is associated.

An embodiment of the PLB problem is the pair $(A, U F)$ in which $A$ is a finite set and $U F: A \rightarrow \mathbb{Z}$ is some function. The aim is to identify a solution $\left[p^{*}\right]$ included in set $A$ that minimizes the UF at the level of the EDS. Thus, the elements of set $A$ represents admissible solutions $U F$ is the objective function, and $\left[p^{*}\right]$ is the optimal solution of the PLB problem. The set $A$ includes a small number of the elements (only three combinations associated with each end-user), and as such, the optimal solution results from listing all these solutions. The complete enumeration operation has been used to determine the optimal solution considering that the number of the admissible solutions is small, see Table 2 .

Table 3 details the stages of the implementation procedure, and Figure 8 presents the flowchart of the proposed centralized control methodology.

Table 2. The admissible solutions associated with the phase swapping of the consumer/prosumers.

\begin{tabular}{ccc}
\hline Consumer/Prosumer Type & Initial positions & Final positions \\
\hline \multirow{2}{*}{$1-\mathrm{P}$ type } & $a$ & $b / c / a$ \\
& $b$ & $c / a / b$ \\
& $c$ & $a / b / c$ \\
\hline
\end{tabular}

The procedure can be used for all strategies applied in an AEDN: 1-P consumerlevel (C-PLB), 1-P prosumer-level (P-PLB), and hybrid-level (H-PLB) for all 1-P endusers (prosumers and consumers). Also, the procedure runs regardless of the status of the SSDs $(\mathrm{On} / \mathrm{Off})$ and the absorbed/injected current (equal/different to 0), the 1-P enduser (prosumer or consumer) being or not considered in the PLB process.

The 3-P prosumers and consumers are treated as the non-switchable end-users. Regarding the 3-P prosumers, they should be equipped with special complex devices based on tracking the values of phase currents and modifying the current injection for balancing at the level of the pole or area. 


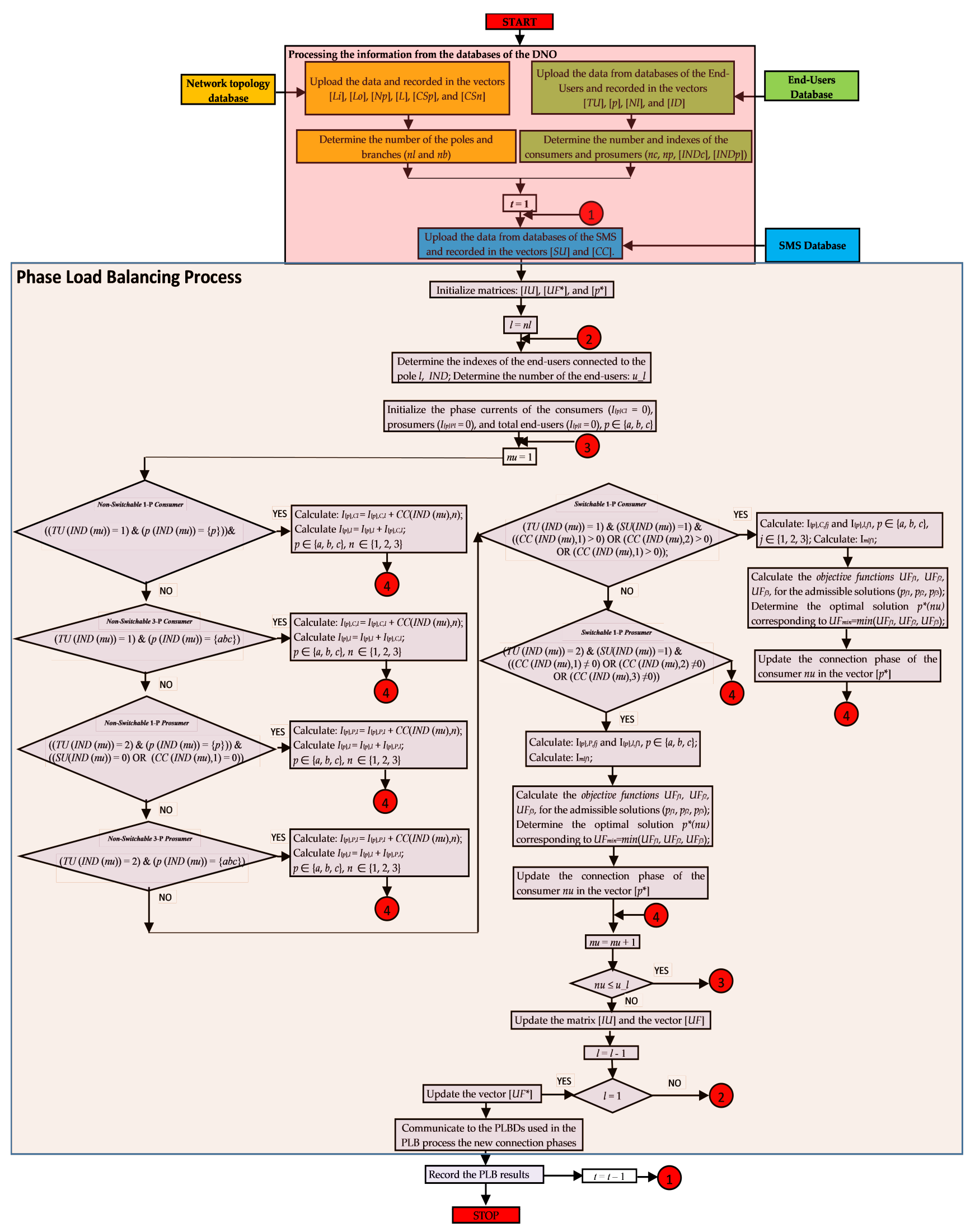

Figure 8. The flow-chart of the proposed coordinated control algorithm of 1-P End-Users for Phase Load Balancing in the AEDNs

Table 3. The implementation procedure of the proposed PLB methodology. 
Stages of the H-PLB methodology based on participation of all end-users (consumers and prosumers)

Stage 1. Identifying the topology of the AEDN.

Upload the data from the topology database and recorded in the vectors $[L],[C S],[N p],[L i]$, and $[L o]$.

Determine from the vector [ $L i]$ the number of the poles: $n l=$ length $(L i)+1$;

Determine from the vector $[\mathrm{Li}]$ the number of the branches: $n b=$ length $(\mathrm{Li})$;

Stage 2. Querying the databases of the DNO (SMS and End-Users)

Upload the data from databases of End-Users and recorded in the vectors [Nl], [p], [ID], and [TU].

Determine from the vector $[\mathrm{TU}]$ the following information:

- the indexes of the consumers: $\operatorname{INDc}=\operatorname{find}([T U]=1)$;

- the number of the consumers: $n c=l e n g t h([I N D c])$;

- the indexes of the prosumers: $I N D p=\operatorname{find}(T U=2)$;

for each hour $t=1: T$

- the number of the prosumers: $n p=$ length $(I N D p)$;

Upload the data from databases of the SMS and recorded in the vectors [SU] and [CC].

Step 3. The PLB process implemented to the level of each pole:

Initialization of the matrix corresponding to the total phase currents of the end-users connected at each pole, [IU], having the size (nlx3), and the vectors referring to the unbalance factor at the level of the EDS, [UF*], and the optimal connection phase of the end-users, $\left[p^{*}\right]$, with the size (Tx1).

Set the index of the pole (starting with the end poles, in descending order): $l=n l$;

while $(l \geq 1) \&(l \leq n l)$

Determine the indexes of the end-users connected to the pole $l: I N D=$ find $([N l]=l)$;

Determine the number of the end-users: $u_{-} l=$ length ([IND]);

Initialize the total phase currents corresponding to:

consumers: $I_{a C l}=0, I_{b C l}=0, I_{c C l}=0$;

prosumers: $I_{a P l}=0, I_{b P l}=0, I_{c P l}=0$;

end-users: $I_{a l}=0, I_{b l}=0, I_{c l}=0$;

Set index of the end-users: $n u=0$;

while $n u \leq u \_l$

Increase index of the end-users: $n u=n u+1$;

case Non-Switchable 1-P Consumer - the end-user $n u$ is a consumer having the SSD with the status Off

or the hourly absorbed phase current with the null value.

if $((\operatorname{TU}(\operatorname{IND}(n u))=1) \&(p(\operatorname{IND}(n u))=\{a\})) \&((\operatorname{SU}(\operatorname{IND}(n u))=0)$ OR $(C C(\operatorname{IND}(n u), 1)=0))$;

Update the total current of the consumers on the phase $a$ :

$I_{a C l}=I_{a C l}+C C(\operatorname{IND}(n u), 1)$;

Calculate the total current on the phase $a$ :

$I_{a l}=I_{a l}+I_{a C l}$;

if $((\operatorname{TU}(\operatorname{IND}(n u))=1) \&(p(\operatorname{IND}(n u))=\{b\})) \&((\operatorname{SU}(\operatorname{IND}(n u))=0)$ OR $(C C(\operatorname{IND}(n u), 2)=0))$;

Update the total current of the consumers on the phase $b$ :

$I b C l=I b c l+C C(I N D(n u), 2)$;

Calculate the total current on the phase $b$ :

$I b l=I b l+I_{b C l}$;

if $((\operatorname{TU}(\operatorname{IND}(n u))=1) \&(p(\operatorname{IND}(n u))=\{c\})) \&((\operatorname{SU}(\operatorname{IND}(n u))=0)$ OR $(C C(\operatorname{IND}(n u), 3)=0))$;

Update the total current of the consumers on the phase $c$ :

$I_{c C l}=I_{c C l}+C C(I N D(n u), 3)$;

Calculate the total current on the phase $c$ :

$I_{c l}=I_{c l}+I_{c C l}$;

case Non-Switchable 3-P Consumer - the end-user $n u$ is a 3-P consumer.

if $(T U(I N D(n u))=1) \&(p(I N D(n u))=\{a b c\})$

Update the total current of the consumers on all phases:

$I_{a C l}=I_{a C l}+C C(\operatorname{IND}(n u), 1) ; I_{b C l}=I_{b C l}+C C(\operatorname{IND}(n u), 2) ; I_{c C l}=I_{c C l}+C C(\operatorname{IND}(n u), 3)$.

Calculate the total current on all phases:

$I_{a l}=I_{a l}+I_{a C l} ; I_{b l}=I_{b l}+I_{b c l} ; I_{c l}=I_{c l}+I_{c c l}$. 
case Non-Switchable 1-P Prosumer - the end-user $n u$ is a prosumer having the SSD with the status Off or the hourly injected/absorbed phase current with the null value.

if $((T U(\operatorname{IND}(n u))=2) \&(p(\operatorname{IND}(n u))=\{a\})) \&((\operatorname{SU}(\operatorname{IND}(n u))=0)$ OR $(C C(\operatorname{IND}(n u), 1)=0))$;

Update the total current of the prosumers on the phase $a$ :

$I_{a P l}=I_{a P l}+C C(I N D(n u), 1)$;

Calculate the total current on the phase $a$ :

$I_{a l}=I_{a l}+I_{a P l} ;$

if $((\operatorname{TU}(\operatorname{IND}(n u))=2) \&(p(\operatorname{IND}(n u))=\{b\})) \&((\operatorname{SU}(\operatorname{IND}(n u))=0)$ OR $(C C(\operatorname{IND}(n u), 2)=0))$;

Update the total current of the prosumers on the phase $b$ :

$I_{b P l}=I_{b P l}+C C(I N D(n u), 2)$;

Calculate the total current on the phase $b$ :

$I b l=I b l+I_{b P l} ;$

if $((\operatorname{TU}(\operatorname{IND}(n u))=2) \&(p(\operatorname{IND}(n u))=\{c\})) \&((\operatorname{SU}(\operatorname{IND}(n u))=0)$ OR $(C C(\operatorname{IND}(n u), 3)=0))$;

Update the total current of the prosumers on the phase $c$ :

$I_{c P l}=I_{c P l}+C C(I N D(n u), 3)$;

Calculate the total current on the phase $c$ :

$I_{c l}=I_{c l}+I_{c P l}$;

case Non-Switchable 3-P Prosumer - the end-user $n u$ is a 3-P prosumer.

if $(T U(\operatorname{IND}(n u))=2) \&(p(I N D(n u))=\{a b c\})$

Update the total current of the prosumers on all phases:

$I_{a P l}=I_{a P l}+C C(\operatorname{IND}(n u), 1) ; I_{b P l}=I_{b P l}+C C(\operatorname{IND}(n u), 2) ; I_{c P l}=I_{c P l}+C C(\operatorname{IND}(n u), 3)$.

Calculate the total current on all phases::

$I_{a l}=I_{a l}+I_{a P l} ; \quad I_{b l}=I_{b l}+I_{b P l} ; \quad I_{c l}=I_{c l}+I_{c P l}$.

case Switchable 1-P Consumer - the end-user $n u$ is a consumer having the SSD with the status On and the hourly absorbed phase current with the positive value.

if $(T U(\operatorname{IND}(n u))=1) \&(\operatorname{SU}(\operatorname{IND}(n u))=1) \&$

$((C C(\operatorname{IND}(n u), 1)>0)$ OR $(C C(\operatorname{IND}(n u), 2)>0)$ OR $(C C(\operatorname{IND}(n u), 1)>0))$;

Identification of the optimal connection solution of the consumer $n u$ using the combinatorial optimization:

Admissible solution 1 - connection on the phase a

Determine the feasible phase currents:

$I_{a C f 1}=I_{a C l}+C C(\operatorname{IND}(n u), 1) ; I_{b C f 1}=I_{b C l} ; I_{c c f 1}=I_{c c l}$;

Determine the feasible total phase currents:

$I_{a l f 1}=I_{a l}+I_{a c f 1} ; \quad I_{b l f 1}=I_{b l}+I_{b c f 1} ; \quad I_{c l f 1}=I_{c l}+I_{c c f 1}$;

Determine the mean of the phase currents, Imlf1 (formula (2))

Determine the $U F_{f 1}$ (formula (1));

Admissible solution 2 - connection on the phase $b$

Determine the feasible phase currents:

$I_{a C f 2}=I_{a C l} ; I_{b C f 2}=I_{b c l}+C C(I N D(n u), 2) ; I_{c c f 2}=I_{c c l}$;

Determine the feasible total phase currents:

$I_{a l f 2}=I_{a l}+I_{a c f 2} ; I_{b l f 2}=I_{b l}+I_{b c f 2} ; I_{c l f 2}=I_{c l}+I_{c c f 2} ;$

Determine the mean of the phase currents, $I_{m l f 2}$ (formula (2))

Determine the $U F_{f 2}$ (formula (1));

Admissible solution 3 - connection on the phase $c$

Determine the feasible phase currents:

$I_{a C \beta}=I_{a C l} ; I_{b C \beta}=I_{b C l} ; I_{c c \beta 3}=I_{c C l}+C C(\operatorname{IND}(n u), 3)$;

Determine the feasible total phase currents:

$I_{a l f 3}=I_{a l}+I_{a C f 3} ; I_{b l f 3}=I_{b l}+I_{b C f 3} ; I_{c l f 3}=I_{c l}+I_{c \subset f 3} ;$

Determine the mean of the phase currents, $I_{m l f 3}$ (formula (2))

Determine the $U F_{f 3}$ (formula (1));

Calculate the optimal solution that leads to the minimization of the $U F: U F_{\min }=\min \left(U F_{f 1}, U F_{f 2}, U F_{f 3}\right)$;

if Optimal solution $=$ Admissible solution 1

Update the vector $\left[p^{*}\right]: p^{*}(\operatorname{IND}(n u))=\{a\}$; 
Update the total phase currents of consumers:

$I_{a C l}=I_{a C f 1} ; I_{b C l}=I_{b C f 1} ; I_{c C l}=I_{c \subset f f} ;$

Update the total phase currents of the end-users:

$I_{a l}=I_{a l f 1} ; I_{b l}=I_{b l f 1} ; I_{c l}=I_{c l f 1} ;$

if Optimal solution $=$ Admissible solution 2

Update the vector $\left[p^{*}\right]: p^{*}(\operatorname{IND}(n u))=\{b\}$;

Update the total phase currents of consumers:

$I_{a C l}=I_{a C f 2} ; I_{b C l}=I_{b c f 2} ; I_{c C l}=I_{c c f 2}$;

Update the total phase currents of the end-users:

$I_{a l}=I_{a l f 2} ; I_{b l}=I_{b l f 2} ; I_{c l}=I_{c l f 2} ;$

else

Update the vector $\left[p^{*}\right]: p^{*}(\operatorname{IND}(n u))=\{c\}$;

Update the total phase currents of prosumers:

$I_{a C l}=I_{a C f 3} ; I_{b C l}=I_{b C 3} ; I_{c C l}=I_{c C F} ;$

Update the total phase currents of the end-users:

$I_{a l}=I_{a l f 3} ; I_{b l}=I_{b l f 3} ; I_{c l}=I_{c l f 3} ;$

case Switchable 1-P Prosumer - the end-user $n u$ is a prosumer having the SSD with the status On and the hourly absorbed/injected phase current with the different value by 0 .

if $(T U(\operatorname{IND}(n u))=2) \&(S U(I N D(n u))=1) \&$ $((C C(\operatorname{IND}(n u), 1) \neq 0)$ OR $(C C(\operatorname{IND}(n u), 2) \neq 0)$ OR $(C C(\operatorname{IND}(n u), 3) \neq 0))$;

Identification of the optimal connection solution of the prosumer $n u$ using the combinatorial optimization:

Admissible solution 1 - connection on the phase a

Determine the feasible phase currents:

$I_{a P f 1}=I_{a P l}+C C(I N D(n u), 1) ; I_{b P f 1}=I_{b P l} ; I_{c P f 1}=I_{c P l} ;$

Determine the feasible total phase currents:

$I_{a l f 1}=I_{a l}+I_{a p f l} ; I_{b l f 1}=I_{b l}+I_{b P f 1} ; I_{c l f 1}=I_{c l}+I_{c p f 1} ;$

Determine the mean of the phase currents, Imlf (formula (2))

Determine the $U F_{f 1}$ (formula (1));

Admissible solution 2 - connection on the phase $b$

Determine the feasible phase currents:

$I_{a P f 2}=I_{a P l} ; I_{b P f 2}=I_{b P l}+C C(I N D(n u), 2) ; I_{c P P 2}=I_{c P l} ;$

Determine the feasible total phase currents:

$I_{a l f 2}=I_{a l}+I_{a P f 2} ; \quad I_{b l f 2}=I_{b l}+I_{b P f 2} ; I_{c l f 2}=I_{c l}+I_{c P f 2} ;$

Determine the mean of the phase currents, $I_{m l f 2}$ (formula (2))

Determine the $U F_{f 2}$ (formula (1));

Admissible solution 3 - connection on the phase $c$

Determine the feasible phase currents:

$I_{a P f 3}=I_{a P l} ; I_{b P f 3}=I_{b P l} ; I_{C P f 3}=I_{c P l}+C C(\operatorname{IND}(n u), 3)$;

Determine the feasible total phase currents:

$I_{a l f 3}=I_{a l}+I_{a P f} ; I_{b l / 3}=I_{b l}+I_{b P f 3} ; I_{c l f 3}=I_{c l}+I_{c P f 3} ;$

Determine the mean of the phase currents, $I_{m l / \beta}$ (formula (2))

Determine the $U F_{f 3}$ (formula (1));

Calculate the optimal solution that leads to the minimization of the $U F: U F_{\min }=\min \left(U F_{f 1}, U F_{f 2}, U F_{f 3}\right)$;

if Optimal solution $=$ Admissible solution 1

Update the vector $\left[p^{*}\right]: p^{*}(\operatorname{IND}(n u))=\{a\}$;

Update the total phase currents of prosumers:

$I_{a P l}=I_{a P f l} ; I_{b P l}=I_{b p f 1} ; I_{c P l}=I_{c P f 1} ;$

Update the total phase currents of the end-users:

$I_{a l}=I_{a l f 1} ; I_{b l}=I_{b l f 1} ; I_{c l}=I_{c l f 1} ;$

if Optimal solution $=$ Admissible solution 2

Update the vector $\left[p^{*}\right]: p^{*}(\operatorname{IND}(n u))=\{b\}$;

Update the total phase currents of prosumers:

$I_{a P l}=I_{a P \rho 2} ; I_{b P l}=I_{b P \rho 2} ; I_{c P l}=I_{c P \rho 2} ;$ 
Update the total phase currents of the end-users:

else

$$
I_{a l}=I_{a l f 2} ; I_{b l}=I_{b l f 2} ; I_{c l}=I_{c l f 2}
$$

Update the vector $\left[p^{*}\right]: p^{*}(\operatorname{IND}(n u))=\{c\}$;

Update the total phase currents of prosumers:

$I_{a P l}=I_{a P \beta} ; I_{b P l}=I_{b P P 3} ; I_{c P l}=I_{c P \rho} ;$

Update the total phase currents of the end-users:

$I_{a l}=I_{a l f 3} ; I_{b l}=I_{b l f 3} ; I_{c l}=I_{c l f 3} ;$

Update the matrix $[I U]: \operatorname{IU}(l, 1)=I_{a l}, I U(l, 2)=I b l$, and $I U(l, 3)=I_{c l}$;

Update the vector $[U F]: U F(l)=U F_{\min }$;

Decrease index of the pole: $l=l-1$;

Update the vector $\left[U F^{*}\right]: U F^{*}(t)=U F(1)$;

Communicate to the PLBDs used in the PLB process the new connection phases;

Record the PLB results in matrix $[P A]$

Increase the index of hour: $t=t+1$;

Print the results.

\section{Case Study}

The proposed methodology has been tested on a LV AEDN belonging to a Romanian DNO with 114 end-users integrated into the Smart Metering System (SMS), including 104 consumers (103 single-phase/ 1 three-phase) and 10 single-phase prosumers, during a day when the peak load has been recorded. The prosumers have the PV panels with the installed power of $1.5,3$, and $5 \mathrm{~kW}$, without storage systems.

The common coupling point (CCP) with the network operated by the DNO is on the low voltage busbar $(0.4 \mathrm{kV})$ of the electric distribution substation. Figure 9 presents the topology of the AEDN, and details of the technical characteristics can be seen in the supplementary file Additional Data of the Topology.

Table 4 synthesizes the information on the number of consumers/prosumers, the length, and cross-sections of the main trunk (MT)/lateral branch (LB).

Table 4. The topology data associated with the analysed AEDN

\begin{tabular}{cccccccc}
\hline \multirow{2}{*}{ AEDN } & $\begin{array}{c}\text { Number } \\
\text { of poles }\end{array}$ & $\begin{array}{c}\text { Pole } \\
\mathbf{i}\end{array}$ & $\begin{array}{c}\text { Pole } \\
\mathbf{j}\end{array}$ & $\begin{array}{c}\text { Length } \\
{[\mathbf{k m}]}\end{array}$ & \multicolumn{2}{c}{ Cross-section [mm2] } & Consumers/ \\
& & CCP & P5 & 0.20 & $3 \times 50$ & 50 & 5 \\
Main & & P5 & P66 & 1.08 & $3 \times 50$ & 50 & $28 / 7$ \\
trunk & \multirow{2}{*}{53} & P66 & P88 & 0.80 & $3 \times 50$ & 50 & $19 / 1$ \\
(MT) & & P66 & P68 & 0.08 & $1 \times 16$ & 25 & 1 \\
& & P5 & P13 & 0.32 & $3 \times 35$ & 35 & 11 \\
Lateral & & P13 & P20 & 0.28 & $1 \times 35$ & 35 & 6 \\
branch & \multirow{2}{*}{35} & P8 & P32 & 0.48 & $3 \times 35$ & 35 & $22 / 2$ \\
(LB) & & P29 & P39 & 0.28 & $1 \times 25$ & 25 & 12 \\
\hline
\end{tabular}




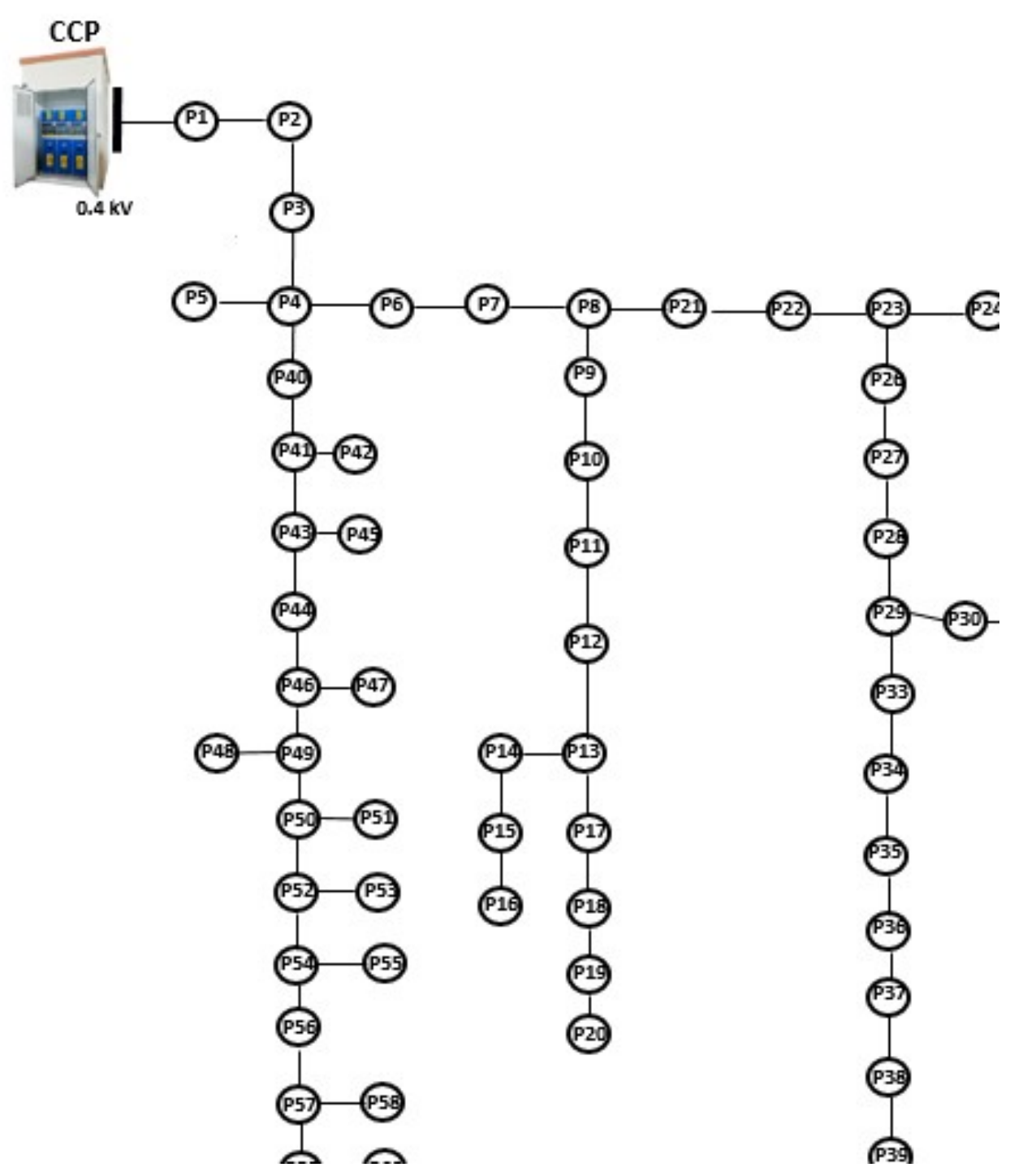

Figure 9. The topology of the test AEDN with 89 poles (including $\mathrm{CCP}$ ) and 88 branches

The AEDN has a main trunk characterized by a total length of $2.16 \mathrm{~km}$, containing 53 poles to which 61 end-users (53 consumers/8 prosumers) are connected. A single lateral branch leaves from the pole P5 having $1.36 \mathrm{~km}$ and 53 end-users (51 consumers/2 prosumers) connected at 35 poles. Also, the wires of the main trunk have a cross-section of $50 \mathrm{~mm}^{2}$, unlike the lateral branch with a cross-section of $35 \mathrm{~mm}^{2}$.

An uneven allocation of the end-users was observed both on the main trunk and the lateral branch, see Table 5 and Figure 10.

Most of the end-users connected at the poles from the main trunk are connected on phase $b(83.6 \%)$ and a smaller number on phases a $(13.1 \%)$ and c $(3.3 \%)$. The lateral branch has a similar situation, with a high allocation on phase c $(75.5 \%)$ and a discrete presence on phases $a(15.1 \%)$ and $b(7.5 \%)$. Only one three-phase end-user $(1.9 \%)$ from the consumers' category, connected on the lateral branch, has been identified in the AEDN.

All data were recorded in the input vectors having the size $(1 \mathrm{x} N c)$, where $N c$ represents the number of the end-users: $[P]$ (number of the pole), $[p]$ (allocated phase), $[L]$ (length of each section), [Ct] (conductor type, single-phase or three-phase), [CSp] (crosssection of the phase conductor), and [CSn] (cross-section of the neutral conductor). The elements of the vectors $[L],[C t],[C S p]$, and $[C S n]$ refer to the information of the section 
between two poles of the main trunk or lateral branch. Also, the vectors allow the Decision Maker to know the detailed topology of the analysed AEDN.

Table 5. Location and connected phase (initial situation) of the end-users in the AEDN

\begin{tabular}{|c|c|c|c|c|c|c|c|c|c|c|c|}
\hline End-User $\mathrm{F}$ & Branch & Pole & Phase & End-User & Branch & Pole & Phase & End-User & Branch & Pole & Phase \\
\hline CONS1 & MT & P1 & Phase b & CONS83 & MT & P63 & Phase $b$ & CONS21 & LB & P20 & Phase c \\
\hline CONS2 & MT & P2 & Phase a & CONS84 & MT & P65 & Phase $b$ & CONS22 & LB & P20 & Phase c \\
\hline CONS3 & MT & P3 & Phase b & CONS85 & MT & P68 & Phase $b$ & CONS23 & LB & P21 & Phase $b$ \\
\hline CONS4 & MT & P4 & Phase $b$ & CONS86 & MT & P70 & Phase $b$ & CONS24 & LB & P21 & Phase c \\
\hline CONS5 & MT & P5 & Phase b & CONS87 & MT & P71 & Phase a & CONS25 & LB & P22 & \\
\hline PROS3 & MT & P40 & Phase b & CONS88 & MT & P71 & Phase $b$ & CONS26 & LB & P22 & Phase c \\
\hline PROS4 & MT & P40 & & CONS89 & & P71 & & CONS27 & LB & P23 & Phase c \\
\hline CONS57 & MT & P41 & Phase a & $\mathrm{CON}$ & MT & P71 & Phase $b$ & CONS28 & LB & P23 & Phase c \\
\hline CONS58 & MT & P41 & Phase $b$ & CONS91 & MT & P72 & Phase $b$ & CONS29 & LB & P24 & \\
\hline CONS59 & MT & P41 & & CONS & MT & P73 & & $\mathrm{CON}$ & & P25 & \\
\hline CONS60 & MT & P42 & ase $b$ & $\mathrm{CON}$ & MT & P75 & $\mathrm{e} b$ & $\mathrm{CON}$ & LB & P26 & \\
\hline CONS61 & $\mathrm{M}$ & & ase $b$ & $\mathrm{CO}$ & & P75 & & & & P26 & \\
\hline CONS62 & MT & & Phase c & & & P76 & se a & & LB & P26 & \\
\hline CONS63 & MT & P45 & & $\mathrm{COI}$ & & P77 & & & LB & P26 & \\
\hline PROS5 & MT & 45 & & $\mathrm{CON}$ & & P80 & & & LB & P27 & \\
\hline CONS64 & MT & & & $\mathrm{CON}$ & & P80 & & & LB & P27 & \\
\hline CONS65 & MT & P47 & & PRO & M & P81 & Pha & COI & LB & P27 & e a \\
\hline & $\mathrm{M}^{\prime}$ & & & & & P82 & & & & P28 & \\
\hline CONS67 & MT & & & $\mathrm{CON}$ & & P82 & & & LB & P28 & \\
\hline PROS6 & MT & P47 & & ON & MT & P85 & & $\mathrm{CO}$ & LB & P28 & \\
\hline PROS7 & MT & & lase $b$ & S012 & MT & P86 & & & LB & P30 & \\
\hline CONS68 & MT & P48 & Phase b & CONS103 & MT & P86 & Phase $b$ & & LB & P30 & \\
\hline PROS8 & MT & & & CON & $M^{\prime}$ & P88 & & & LB & P30 & \\
\hline CONS69 & MT & & tase $b$ & & $\mathrm{Ll}$ & P6 & Phase $b$ & $\mathrm{CO}$ & LB & P31 & \\
\hline CONS70 & MT & P52 & Phase b & $\mathrm{CON}$ & LE & P6 & Phase c & $\mathrm{COI}$ & LB & P32 & Phase c \\
\hline CONS71 & MT & P53 & Phase b & CONS8 & LB & P7 & Phase c & $\mathrm{COI}$ & LB & P32 & Phase c \\
\hline CONS72 & MT & & Phase c & CONS9 & LB & P8 & Phase $b$ & CONS45 & LB & P33 & Phase c \\
\hline CONS73 & MT & P56 & Phase a & CONS10 & LB & P9 & Phase c & CONS46 & LB & P33 & Phase c \\
\hline & MT & & & & & P10 & Phase abc & & & P34 & \\
\hline CONS75 & MT & & Phase a & CONS12 & LB & P11 & Phase c & CON & LB & P35 & Phase c \\
\hline CONS76 & MT & P59 & Phase b & CONS13 & LB & P11 & Phase c & CONS49 & LB & P36 & Phase c \\
\hline PROS9 & MT & P59 & Phase b & CONS14 & LB & P12 & Phase a & CONS50 & LB & P36 & Phase c \\
\hline CONS77 & MT & P60 & Phase $b$ & CONS15 & LB & P13 & Phase a & CONS51 & LB & P37 & Phase c \\
\hline CONS78 & MT & P60 & Phase b & CONS16 & LB & P13 & & CONS52 & LB & P37 & Phase $b$ \\
\hline CONS79 & MT & P61 & & & LB & P16 & & & LB & P38 & Phase c \\
\hline CONS80 & MT & P61 & Phase b & CONS18 & LB & P16 & Phase a & CONS54 & LB & P38 & Phase c \\
\hline CONS81 & MT & P62 & Phase a & CONS19 & LB & P19 & Phase c & CONS55 & LB & P38 & Phase c \\
\hline CONS82 & MT & P62 & Phase b & CONS20 & LB & P19 & Phase c & CONS56 & LB & P39 & Phase c \\
\hline
\end{tabular}

*MT - Main trunck; LB - Lateral Branch; CONS - consumer; PROS - prosumer. 


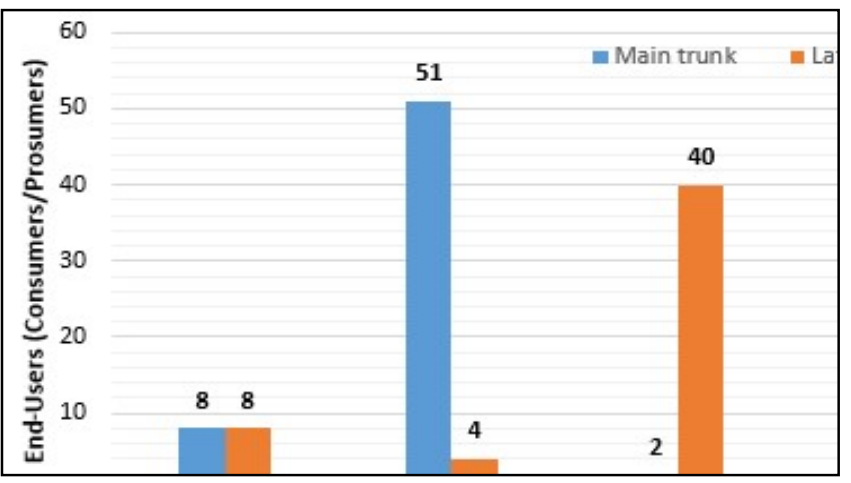

Figure 10. The type of the end-users (consumers/prosumers) and their allocation on the phases of the analysed AEDN

Additional data referring to the hourly current of the consumers and the injected/absorbed current of the prosumers are known in real-time. The processing module queried the SMS' database and uploaded the recorded values of each end-user from the analyzed AEDN in the vectors $[I C]$, for the consumers, and $[I P]$, for the prosumers. The current profiles are available in the supplementary file End-Users' Current Profiles.

After data uploading, the next step has been initialized to obtain the aggregate value of the phase powers at the CCP level and calculate the unbalance factor. Figures 11 and 12 show the hourly values of the aggregated phase currents and the UF at the CCP level in the initial case, without PLB (W-PLB).

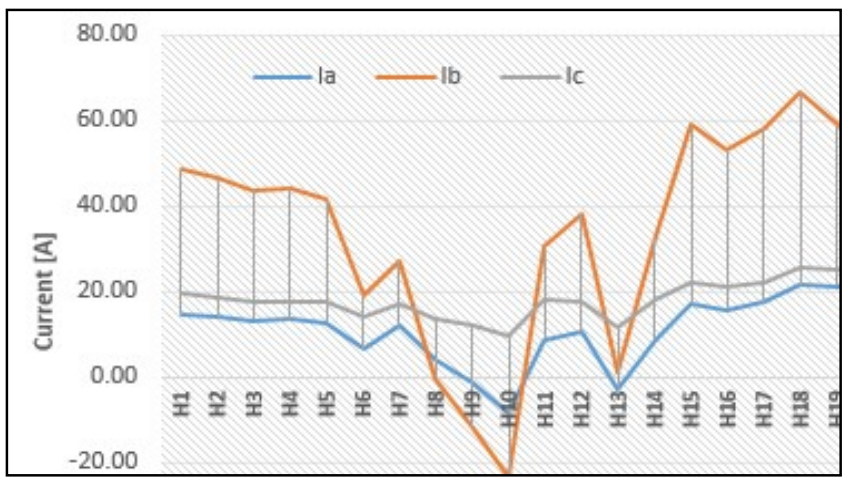

Figure 11. The aggregated phase currents at the level of the CCP (W-PLB case)

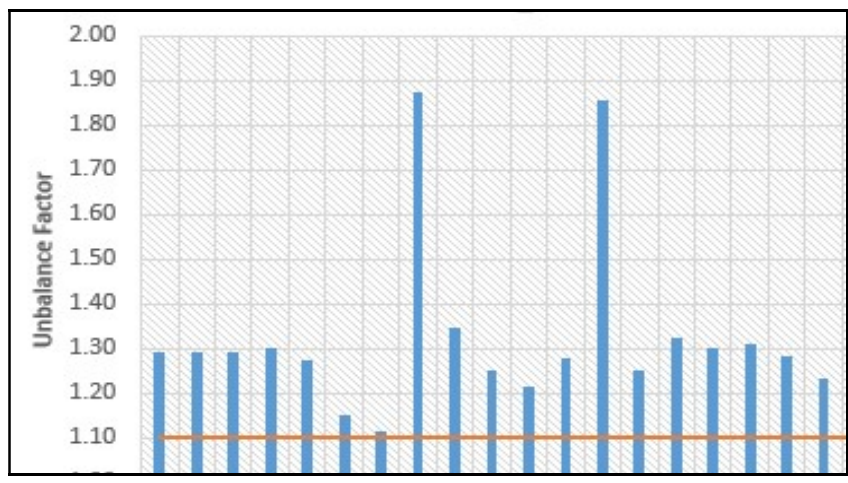

Figure 12. The hourly unbalance factor obtained at the CCP level (W-PLB case) 
Inverse current flows from AEDN to CCP on phases $a$ and $b$, at hours $\mathrm{H} 9$ and $\mathrm{H} 10$, and phase $a$, at hour $\mathrm{H} 13$, have been recorded, which means that prosumers have higher current injections than the demands of consumers connected on these phases. Also, high differences between the phase currents recorded at each hour, leading to values of the UF at every hour over the target accepted by the DNO (UF $=1.1$, corresponding to a degree of current unbalance of $10 \%$ ), represented in brown in the figure. The maximum values of the UF have been recorded at the hours H8 and H13 (1.87 and 1.86), and the average value reached 1.30 .

In these conditions, the steps to establish a phase load balancing in the analysed AEDN, having as objective obtaining the hourly UFs below the target value imposed by the DNO (1.1) at the CCP level, have been completed. The poles located at the end of the network represent the points where the algorithm initialized, and the last point corresponds to the CCP, where the UF must have the minimum value subject to the constraints (2) - (8). The aggregation of the injected/absorbed currents is done in each point, identified through a pole, and the locally optimal solution corresponds to the combination associated with the phase allocations of the end-users that leads to the minimum value of the UF. Each solution is recorded in the matrix $[P A]$ on the column assigned to the hour when the PLB process is ongoing and the row corresponding to each end-user allocated to the pole. All elements associated with matrix $[P A]$ are available in the supplementary file Switching Operation Matrix. Figure 13 presents the switching operations of the SSDs installed to the end-users in the analysed period ( 24 hours).

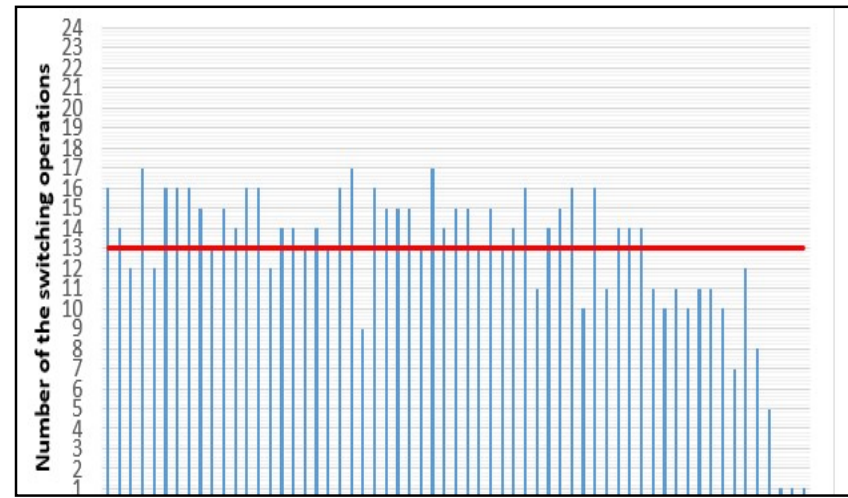

a.

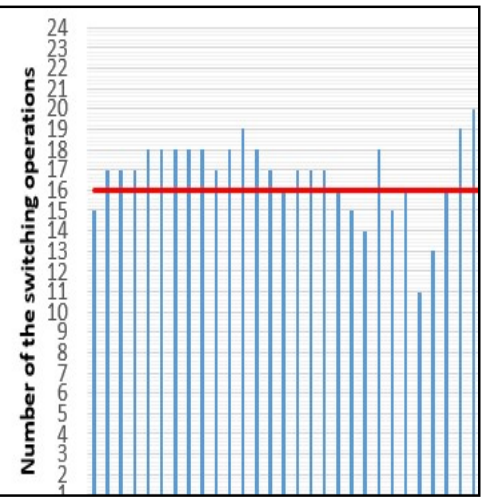

b.

Figure 13. The number of switching operation performed by SSDs ( $\mathrm{a}$ - main trunk; $\mathrm{b}$ - lateral branch

The analysis of the optimal solutions associated with the connection phase at each hour, and recorded in the matrix $[P A]$, relived that each SSD installed at the end-users connected at the poles on the main trunk had on average 13 switching operations and 16 for each SSD on the lateral branch, highlighted with the red line in Figure 12. From all consumers, the maximum number of switching operations (20) has been performed by CONS35 (pole P27, on the lateral branch). The prosumers PROS1 (pole P30), on the lateral branch, PROS3 (pole P40), PROS4 (pole P40), and PROS 7 (pole P47), on the main trunk, had the maximum number of the switching operations (16). These switching operations can be easily supported due to the high reliability associated with the components of the SSDs.

The number of end-users who participated in the PLB process at each hour was different. The maximum number has been recorded at the hour H16 (90 end-users) and the minimum at the hour H3 (31 end-users). The participation degree at the PLB process was as average by $60 \%$ (68 end-users) per hour, see Figure 14 (red line).

The algorithm has been implemented in Matlab2016, the time computing obtained for the optimal solution was 1.13 seconds. The performance is better than that obtained in reference [15] (the computation time was 1.26 seconds), where a similar heuristic PLB 
algorithm has been used and ran on an EDN having the same topology. The same technical characteristics have been considered for the computer (Intel Core i7 processor, 3.10 $\mathrm{GHz}, 4 \mathrm{~GB} \mathrm{RAM}$ ) to perform this comparison.

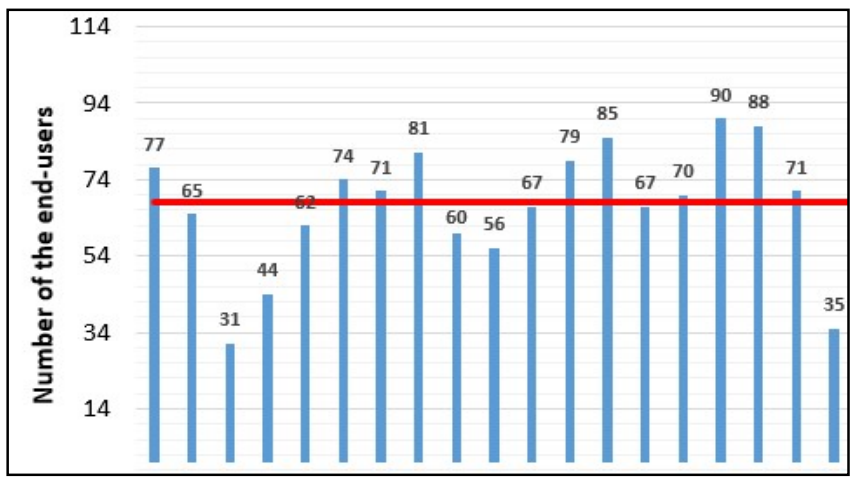

Figure 14. The number of end-users who participated in the PLB process

Figure 15 presents the value of the objective function represented by the minimum value of the UF at the CCP level on the LV side. The average value of the UF is 1.000043 .

The highest values of the UF belong to the hours H8, H9, and H13 (same hours as in the unbalance case), but these are smaller, close by 1.00 (the ideal values). Compared with the unbalance case, the phase currents at the level of the CCP have very close, and there are no inverse current flows from the AEDN to the CCP, see Figure 16.

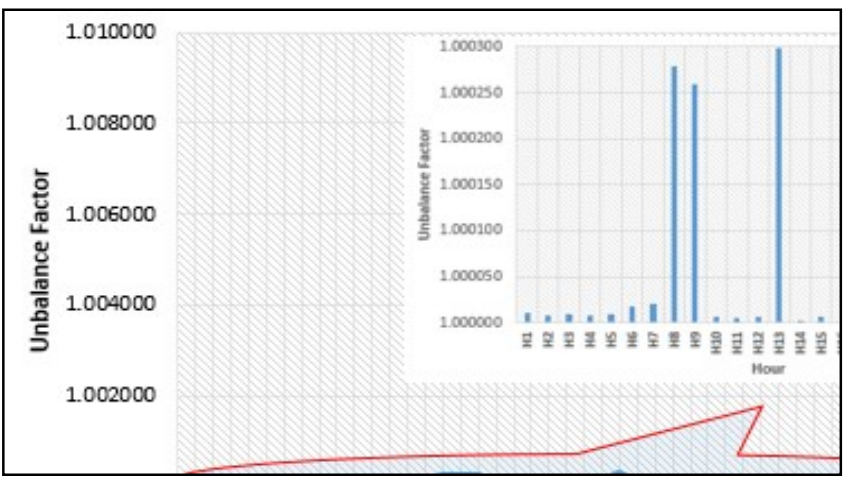

Figure 15. The hourly unbalance factor obtained at the CCP level with the proposed methodology, balanced case

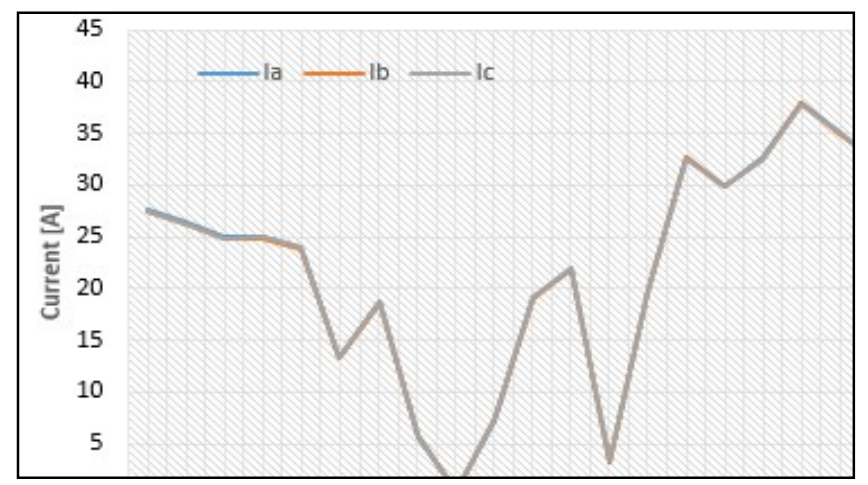

Figure 16. The aggregated phase currents at the level of the CCP obtained with the proposed methodology, H-PLB case

Also, the absorbed phase currents from the CCP at the hour H9 have the smallest values, close by 0 , with a balance of the injected/absorbed currents in the network. 
The other two PLB cases (frequently encountered in the EDNs), applied to the consumer-level (PLB-C) and prosumer-level (PLB-P), have been analysed and compared with the H-PLB case, see Figure 17.

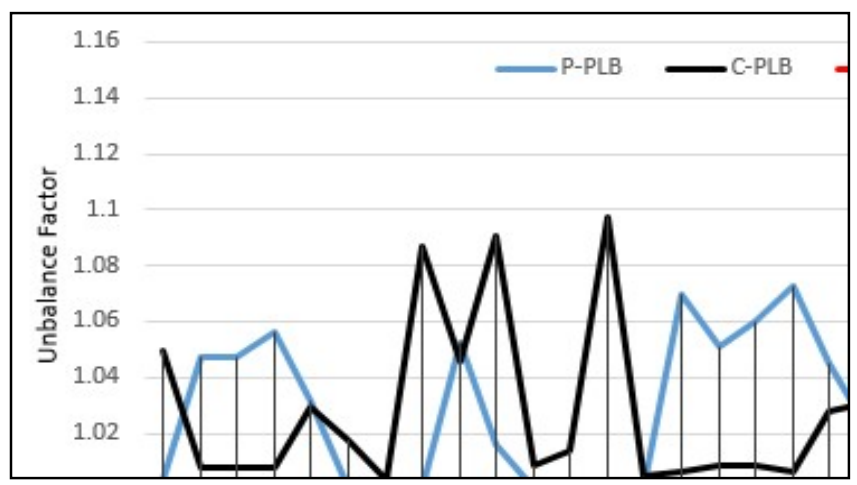

Figure 17. Comparison between the hourly UF obtained in the cases P-PLB, C-PLB, and H-PLB

An analysis of the results led to the following observations regarding the hourly values of the UF. In the P-PLB procedure, two-time intervals, H1 - H5 and H15 - H24, have been identified, where the UF had the higher average values (1.037 and 1.04, respectively). Also, a small average value of 1.008 has been obtained in the time interval H6 - H14. In the C-PLB procedure, the results were the opposite. The UF had the higher values in the time interval H6 - H14 (1.041) and smaller values (1.02 and 1.017) in the other two intervals ( $\mathrm{H} 1$ - H5 and $\mathrm{H} 15$ - H24).

Figure 18 shows the average value of the UF in the three analysed PLB cases. The PPLB and C-PLB cases recorded similar values of the UF (1.028 vs. 1.027), which were higher than in the H-PLB. However, they were below 1.1 (target imposed by the DNO) with approximately 0.07 , emphasizing the effectiveness of the proposed PLB algorithm in these two extreme cases. These positive effects were reflected on the values of the phase currents at the level of the CCP.

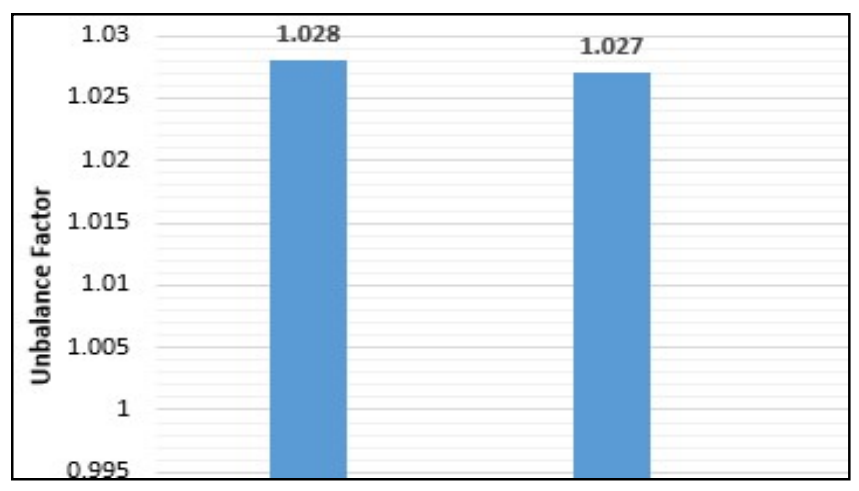

Figure 18. Comparison between the average values of UF calculated in the analysed PLB cases

Figures 19 and 20 detail the results where the time intervals above highlighted can be better observed. There is still an inverse current flow in all phases at the hour H10 in the P-PLB case, but the values are smaller than in the unbalanced case (W-PLB). These current flows did not record in the C-PLB case due to the high number of combinations associated with the consumers. The optimal solutions, characterized through a balance between the injected/absorbed currents by the prosumers and consumers, led to the small phase load unbalances in the C-PLB case. 


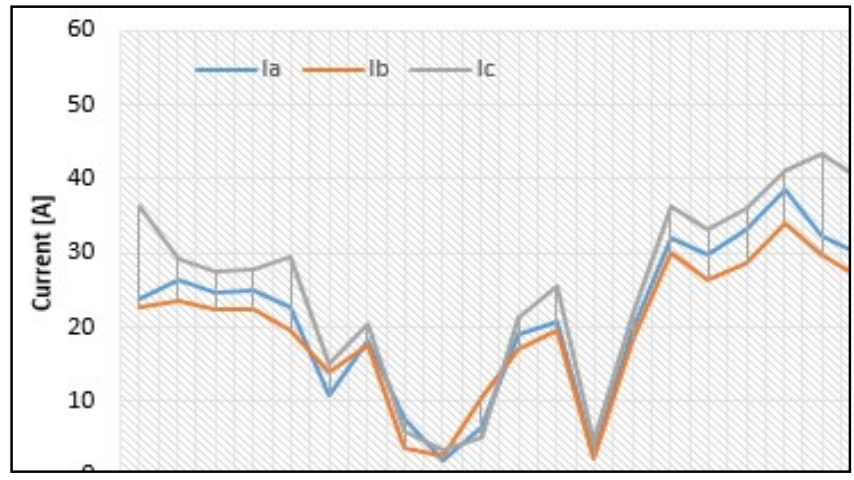

Figure 19. The aggregated phase currents at the level of the CCP obtained with the proposed methodology, P-PLB case

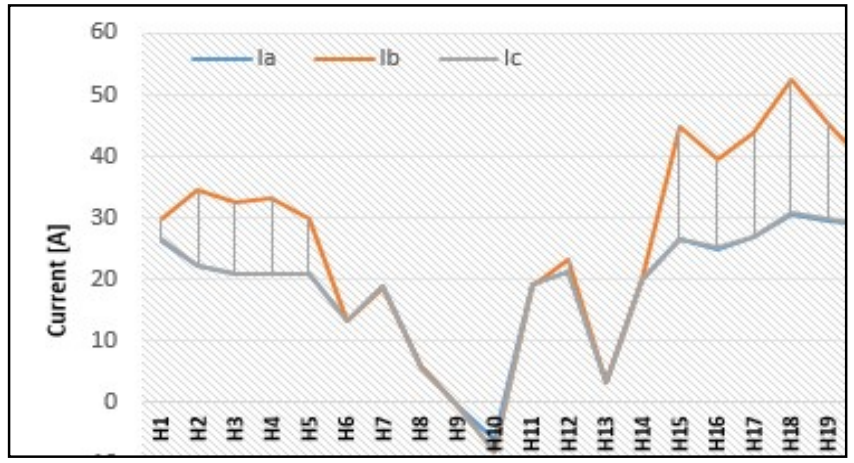

Figure 20. The aggregated phase currents at the level of the CCP obtained with the proposed methodology, C-PLB case

Table 6 contains details regarding the hourly values of the phase currents and UF at the level of the CCP obtained in all analysed cases: without PLB (W-PLB), P-PLB, C-PLB, and H-PLB.

In the final stage, the quantification of the technical benefits obtained after applying the PLB algorithm has been done through energy savings and the phase voltage unbalance rate at the end poles (P88 and P39).

The energy-saving has been calculated based on the following formula:

$$
E S_{P L B}=\left|\frac{\Delta W_{W-P L B}-\Delta W_{P L B}}{\Delta W_{W-P L B}}\right| \cdot 100, \quad[\%]
$$

where: $\triangle W$ PLB represents the energy losses calculated after the application of the PLB algorithm for the cases P-PLB, C-PLB, and H-PLB; $\triangle W_{W-P L B}$ relates to the energy losses calculated for the initial situation, without PLB (W-PLB).

The energy losses have been calculated with an efficient steady-state algorithm based on the forward/backward sweep procedure proposed in [38] for the balanced and unbalanced AEDNs with four wires.

Table 7 and Figure 21 present a comparison between the PLB algorithm considering the average value of the unbalance factor $\left(U F_{a v}\right)$, the energy losses $(\Delta W)$, and energysaving $\left(E S_{P L B}\right)$.

Table 6. The hourly phase currents and the unbalance factor calculated for all analysed cases*, in [kW] 


\begin{tabular}{|c|c|c|c|c|c|c|c|c|c|c|c|c|c|c|c|c|}
\hline \multirow{2}{*}{ Hour } & \multicolumn{4}{|c|}{ W-PLB } & \multicolumn{4}{|c|}{ P-PLB } & \multicolumn{4}{|c|}{ C-PLB } & \multicolumn{4}{|c|}{ H-PLB } \\
\hline & $\mathbf{I}_{\mathbf{a}}$ & $\mathbf{I}_{\mathbf{b}}$ & $I_{c}$ & UF & $I_{a}$ & $I_{b}$ & $I_{c}$ & UF & $\mathbf{I}_{\mathbf{a}}$ & $\mathbf{I b}$ & $I_{c}$ & JF & $I_{a}$ & $I_{b}$ & $\mathbf{I}_{\mathbf{c}}$ & UF \\
\hline H1 & 14.75 & & 19.44 & & 26.36 & 29.86 & 5.46 & 1.003 & 23.69 & 22.78 & 36.20 & 1.049 & 27.68 & 27.51 & 7.47 & 1 \\
\hline $\mathrm{H} 2$ & .99 & 6.35 & 18.61 & 29 & & 34.44 & .25 & 1.048 & .27 & .56 & & & 5.42 & .24 & .29 & \\
\hline H3 & 3.22 & 43.63 & 17.70 & .29 & 20.99 & 32.52 & 1.04 & 1.048 & 24.57 & 22.34 & 27.63 & 1.008 & 24.96 & 24.78 & 4.81 & . 00001 \\
\hline $\mathrm{H} 4$ & 13.35 & 44.21 & 17.42 & 1.30 & 20.79 & 33.37 & 0.81 & 1.056 & 24.91 & 22.28 & 27.79 & 1.008 & 25.09 & 24.94 & 4.95 & 00001 \\
\hline H5 & 12.76 & 41.49 & 17.60 & 1.27 & 20.93 & 29.99 & 0.92 & 1.032 & .71 & 19.65 & 29.49 & 1.029 & 3.95 & 3.86 & 4.04 & 1.00001 \\
\hline H6 & & 1 & .24 & & & 13.24 & & & & .91 & 09 & & & .38 & & \\
\hline H7 & 11.84 & 27.22 & & 12 & & 18.66 & 80 & 1.000 & & 17.73 & 20.30 & & 18.81 & 3.64 & & \\
\hline H8 & & -0.38 & & & & 5.87 & 64 & & & 3.63 & 5.88 & & TI & & 87 & \\
\hline H9 & -1 & 11 & 12.12 & & & -0.28 & -0.39 & & & & & & & & & \\
\hline H10 & & & & & & -7.99 & & & & & & & & & & \\
\hline H11 & 65 & 30.45 & 18.25 & 1.22 & & 19.08 & 9.12 & 1.000 & & 17 & & & & & & \\
\hline H12 & 10.59 & 37.93 & 17.34 & 1.28 & 21.1 & 23.35 & 21.36 & 1.002 & 20. & 19.48 & 25.54 & 1.014 & .90 & 2.03 & 94 & 0001 \\
\hline H13 & -2.81 & 0.84 & 11.79 & 1.86 & 3.26 & 3.20 & .36 & 1.000 & & 2.20 & 4.65 & .097 & 27 & & 35 & 1.00030 \\
\hline H14 & 8 & 32.81 & 18.05 & 1.25 & 19.78 & 19.84 & 19.86 & 1.000 & 19.54 & 18.29 & 21.65 & 1.005 & 19.82 & 19.79 & 19.86 & 1.00000 \\
\hline & 17.25 & 58.91 & 21 & 1.33 & 26.5 & 44.87 & 26.62 & 070 & 3 & 29.97 & 36.16 & 1.006 & 32.80 & .62 & 2.61 & 1.00001 \\
\hline & 15.64 & 52 & & 30 & & 39.38 & .21 & 51 & & 5.50 & 33.26 & .009 & .83 & .89 & .87 & 0000 \\
\hline H17 & & $\pi$ & & & & & & & & & & & & & & \\
\hline H18 & 21.5 & 66.4 & & 29 & & 52.35 & & & & & & & & & & 1.00000 \\
\hline H19 & 21.27 & 58.94 & & 1.23 & & 45.66 & 29.91 & & & 29.74 & 43.28 & & 35.19 & .98 & 5.12 & 1.00001 \\
\hline $\mathrm{H} 20$ & 21.23 & 51.61 & 23.72 & 1.18 & 28.91 & 38.88 & 28.77 & 1.022 & 29.83 & 26.74 & 39.98 & 1.031 & 32.11 & 32.25 & 32.20 & 1.00000 \\
\hline H21 & 25.60 & 58.45 & 27.01 & 1.17 & 32.91 & 45.02 & 33.13 & 1.023 & 40.88 & 30.87 & 39.32 & 1.014 & 36.86 & 37.18 & 37.03 & 1.00001 \\
\hline $\mathrm{H} 22$ & 27.62 & 68.09 & 31.47 & 1.19 & 38.28 & 50.47 & 38.43 & 1.018 & 40.18 & 35.07 & 51.94 & 1.028 & 42.56 & 42.24 & 42.38 & 1.00001 \\
\hline $\mathrm{H} 23$ & 24.7 & 5.12 & 30.58 & 1.22 & & 49.44 & 37.33 & & 39.93 & 35.23 & 48.90 & 1.019 & 41.25 & 41. & 41.58 & 1.00002 \\
\hline H24 & 17.09 & 52.92 & 23.12 & 1.25 & 27.59 & 37.86 & 27.67 & 1.024 & 29.00 & 27.08 & 37.05 & 1.019 & 30.91 & 30.99 & 31.22 & 1.00002 \\
\hline
\end{tabular}

*W-PLB: without PLB; P-PLB: prosumer-level PLB; C-PLB: consumer-level PLB; H-PLB: hybrid (all prosumers and consumers-level) PLB;

Table 7. Comparison between the technical indicators of the AEDN in the analysed cases

\begin{tabular}{lcccc}
\hline \multirow{2}{*}{ Case } & \multirow{2}{*}{ UF $_{\text {av }}$} & \multirow{2}{*}{$\boldsymbol{\Delta W}$} & \multicolumn{2}{c}{ ES } \\
\cline { 4 - 5 } & & {$[\mathbf{k W h}]$} & {$[\mathbf{k W h}]$} & {$[\%]$} \\
\hline W- PLB & 1.260 & 21.52 & - & - \\
P - PLB & 1.028 & 11.90 & 9.62 & 44.70 \\
C - PLB & 1.027 & 10.37 & 11.15 & 51.82 \\
H - PLB & 1.00004 & 8.88 & 12.64 & 58.73 \\
\hline
\end{tabular}

The highest benefits have been recorded using the proposed H-PLB algorithm quantified through an energy-saving of $12.64 \mathrm{kWh}$ in an analysed interval (24 hours), representing $58.73 \%$ of the energy losses calculated in the initial situation (without PLB). The value is higher with $6.91 \%$ than the C-PLB algorithm and $14.03 \%$ compared with the P-PLB algorithm. Table 8 contains details on the hourly total power losses calculated for all analysed cases. 


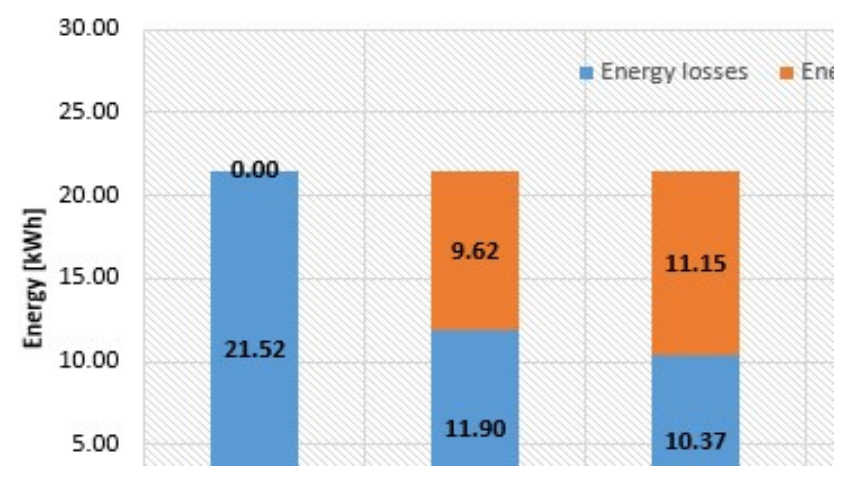

Figure 21. The aggregated phase currents at the level of the CCP obtained with the proposed methodology, P-PLB case

Table 8. The hourly total power losses calculated for all analysed cases, in [kW]

\begin{tabular}{cccccccccc}
\hline \multirow{2}{*}{ Hour } & \multicolumn{4}{c}{ PLB cases } & \multicolumn{3}{c}{ Hour } & \multicolumn{4}{c}{ PLB cases } \\
& W-PLB & P-PLB & C-PLB & H-PLB & & W-PLB & P-PLB & C-PLB & H-PLB \\
\hline H1 & 0.89 & 0.46 & 0.44 & 0.37 & H13 & 0.19 & 0.18 & 0.15 & 0.10 \\
H2 & 0.81 & 0.43 & 0.37 & 0.32 & H14 & 0.50 & 0.27 & 0.29 & 0.22 \\
H3 & 0.72 & 0.37 & 0.33 & 0.29 & H15 & 1.28 & 0.67 & 0.56 & 0.49 \\
H4 & 0.73 & 0.38 & 0.33 & 0.29 & H16 & 1.05 & 0.55 & 0.48 & 0.42 \\
H5 & 0.66 & 0.34 & 0.31 & 0.27 & H17 & 1.26 & 0.67 & 0.56 & 0.50 \\
H6 & 0.23 & 0.11 & 0.16 & 0.10 & H18 & 1.69 & 0.91 & 0.75 & 0.67 \\
H7 & 0.40 & 0.19 & 0.24 & 0.18 & H19 & 1.40 & 0.75 & 0.64 & 0.57 \\
H8 & 0.20 & 0.19 & 0.14 & 0.09 & H20 & 1.14 & 0.59 & 0.55 & 0.49 \\
H9 & 0.27 & 0.33 & 0.15 & 0.10 & H21 & 1.50 & 0.79 & 0.72 & 0.65 \\
H10 & 0.42 & 0.52 & 0.22 & 0.18 & H22 & 2.03 & 1.04 & 0.98 & 0.87 \\
H11 & 0.47 & 0.33 & 0.29 & 0.21 & H23 & 1.99 & 0.99 & 0.91 & 0.83 \\
H12 & 0.58 & 0.28 & 0.30 & 0.23 & H24 & 1.12 & 0.56 & 0.51 & 0.45 \\
\hline
\end{tabular}

The phase voltage unbalance rate (PVUR) has been evaluated according to the IEEE definition [40], [41]:

$$
\operatorname{PVUR}=\frac{\Delta V_{\max }}{V_{a v}} \cdot 100, \quad[\%]
$$

where: $V_{a v}$ is the average value of the phase voltage, and $\Delta V_{\max }$ represents the maximum voltage deviation from the average value of the phase voltage.

Figure 22 and Table 9 present the average value of the PVUR in the analysed interval (24 hours) at the level of each pole.

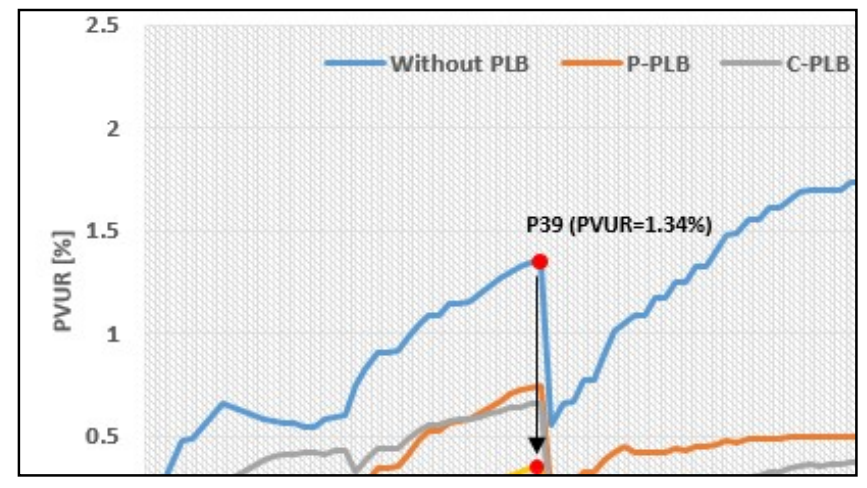

Figure 22. The variation of the PVUR at the level of poles before and after applying the PLB process 
Table 9. The average values of the PVUR calculated at the level of each pole from in the analysed cases, in [\%]

\begin{tabular}{|c|c|c|c|c|c|c|c|c|c|}
\hline \multirow{2}{*}{ Pole } & \multicolumn{4}{|c|}{ PLB cases } & \multirow{2}{*}{ Pole } & \multicolumn{4}{|c|}{ PLB cases } \\
\hline & W-PLB & P-PLB & C-PLB & H-PLB & & W-PLB & P-PLB & C-PLB & H-PLB \\
\hline P1 & 0.11 & 0.03 & 0.02 & 0.01 & P45 & 0.90 & 0.38 & 0.12 & 0.10 \\
\hline P2 & 0.23 & 0.07 & 0.05 & 0.01 & P46 & 1.01 & 0.42 & 0.13 & 0.11 \\
\hline P3 & 0.34 & 0.10 & 0.07 & 0.02 & P47 & 1.05 & 0.45 & 0.14 & 0.12 \\
\hline P4 & 0.48 & 0.15 & 0.10 & 0.03 & P48 & 1.09 & 0.42 & 0.14 & 0.11 \\
\hline P5 & 0.48 & 0.15 & 0.10 & 0.03 & P49 & 1.09 & 0.42 & 0.14 & 0.11 \\
\hline P6 & 0.54 & 0.12 & 0.15 & 0.04 & P50 & 1.17 & 0.42 & 0.16 & 0.11 \\
\hline P7 & 0.60 & 0.11 & 0.21 & 0.05 & P51 & 1.17 & 0.42 & 0.16 & 0.11 \\
\hline P8 & 0.66 & 0.14 & 0.27 & 0.07 & P52 & 1.25 & 0.44 & 0.19 & 0.12 \\
\hline P9 & 0.64 & 0.16 & 0.30 & 0.07 & P53 & 1.25 & 0.43 & 0.19 & 0.12 \\
\hline P10 & 0.62 & 0.18 & 0.33 & 0.07 & P54 & 1.32 & 0.45 & 0.22 & 0.12 \\
\hline P11 & 0.61 & 0.20 & 0.36 & 0.07 & P55 & 1.32 & 0.45 & 0.22 & 0.12 \\
\hline P12 & 0.58 & 0.22 & 0.38 & 0.07 & P56 & 1.40 & 0.46 & 0.24 & 0.13 \\
\hline P13 & 0.57 & 0.23 & 0.40 & 0.07 & P57 & 1.48 & 0.47 & 0.27 & 0.14 \\
\hline P14 & 0.56 & 0.24 & 0.41 & 0.07 & P58 & 1.49 & 0.47 & 0.28 & 0.14 \\
\hline P15 & 0.56 & 0.24 & 0.41 & 0.07 & P59 & 1.55 & 0.49 & 0.30 & 0.15 \\
\hline P16 & 0.55 & 0.24 & 0.42 & 0.07 & P60 & 1.56 & 0.49 & 0.30 & 0.15 \\
\hline P17 & 0.55 & 0.24 & 0.42 & 0.07 & P61 & 1.61 & 0.49 & 0.32 & 0.15 \\
\hline P18 & 0.58 & 0.24 & 0.42 & 0.07 & P62 & 1.61 & 0.49 & 0.32 & 0.15 \\
\hline P19 & 0.60 & 0.25 & 0.43 & 0.08 & P63 & 1.65 & 0.49 & 0.34 & 0.15 \\
\hline P20 & 0.60 & 0.25 & 0.43 & 0.08 & P64 & 1.69 & 0.50 & 0.36 & 0.15 \\
\hline P21 & 0.74 & 0.20 & 0.33 & 0.09 & P65 & 1.69 & 0.50 & 0.36 & 0.15 \\
\hline P22 & 0.83 & 0.27 & 0.38 & 0.11 & P66 & 1.69 & 0.50 & 0.36 & 0.15 \\
\hline P23 & 0.91 & 0.35 & 0.44 & 0.14 & P67 & 1.70 & 0.50 & 0.36 & 0.15 \\
\hline P24 & 0.91 & 0.35 & 0.44 & 0.14 & P68 & 1.70 & 0.50 & 0.36 & 0.15 \\
\hline P25 & 0.91 & 0.35 & 0.44 & 0.14 & P69 & 1.73 & 0.50 & 0.37 & 0.15 \\
\hline P26 & 0.98 & 0.41 & 0.49 & 0.16 & P70 & 1.73 & 0.50 & 0.37 & 0.15 \\
\hline P27 & 1.04 & 0.47 & 0.52 & 0.18 & P71 & 1.77 & 0.50 & 0.38 & 0.15 \\
\hline P28 & 1.09 & 0.52 & 0.55 & 0.20 & P72 & 1.79 & 0.50 & 0.39 & 0.15 \\
\hline P29 & 1.09 & 0.52 & 0.55 & 0.20 & P73 & 1.79 & 0.50 & 0.39 & 0.15 \\
\hline P30 & 1.14 & 0.57 & 0.57 & 0.22 & P74 & 1.82 & 0.51 & 0.39 & 0.16 \\
\hline P31 & 1.15 & 0.57 & 0.58 & 0.23 & P75 & 1.82 & 0.51 & 0.40 & 0.16 \\
\hline P32 & 1.15 & 0.58 & 0.58 & 0.23 & P76 & 1.84 & 0.51 & 0.40 & 0.16 \\
\hline P33 & 1.19 & 0.61 & 0.60 & 0.25 & P77 & 1.85 & 0.51 & 0.40 & 0.16 \\
\hline P34 & 1.23 & 0.64 & 0.61 & 0.27 & P78 & 1.86 & 0.51 & 0.40 & 0.16 \\
\hline P35 & 1.26 & 0.67 & 0.62 & 0.29 & P79 & 1.86 & 0.51 & 0.40 & 0.16 \\
\hline P36 & 1.30 & 0.70 & 0.64 & 0.31 & P80 & 1.88 & 0.51 & 0.40 & 0.16 \\
\hline P37 & 1.33 & 0.73 & 0.64 & 0.33 & P81 & 1.89 & 0.51 & 0.40 & 0.16 \\
\hline P38 & 1.34 & 0.74 & 0.66 & 0.34 & P82 & 1.89 & 0.51 & 0.41 & 0.17 \\
\hline P39 & 1.34 & 0.74 & 0.66 & 0.34 & P83 & 1.89 & 0.51 & 0.41 & 0.17 \\
\hline $\mathrm{P} 40$ & 0.55 & 0.22 & 0.10 & 0.03 & P84 & 1.90 & 0.51 & 0.41 & 0.17 \\
\hline P41 & 0.66 & 0.27 & 0.10 & 0.04 & P85 & 1.90 & 0.51 & 0.41 & 0.17 \\
\hline P42 & 0.67 & 0.27 & 0.10 & 0.04 & P86 & 1.90 & 0.51 & 0.42 & 0.17 \\
\hline P43 & 0.78 & 0.33 & 0.11 & 0.07 & P87 & 1.90 & 0.51 & 0.42 & 0.17 \\
\hline P44 & 0.78 & 0.33 & 0.11 & 0.07 & P88 & 1.91 & 0.51 & 0.42 & 0.17 \\
\hline
\end{tabular}

The highest decrease of the PVUR has been obtained in the case of the H-PLB algorithm. The PUVR has been improved from $1.34 \%$ to $0.34 \%$, and from $1.91 \%$ to $0.17 \%$, respectively, at the level of the end poles, P39 and P88. 


\section{Conclusions}

The AEDNs integrate a high number of 1-P consumers, with a dynamic variation of the demand, and 1-P prosumers with different generation technologies, connected to the three phases, so that the phase currents are no longer equal, leading to current unbalance. Their effects can conduct to the inefficient network utilization, higher losses, higher neutral current, exceeding the allowable limits of the phase-neutral and phase-phase voltages, or difficulties in the voltage control.

The paper concentrated on the phase swapping measure, proposing an original centralized control methodology of the SSDs installed to all 1-P end-users to provide a fast PLB process in the AEDNs. The control of SSDs is done through a communication interface between the end-user and the network. The methodology allows the real-time query simultaneous of three different databases (Topology Database, SMS Database, and EndUsers Database) of the DNO uploading the input data for a fast algorithm applied for all cases encountered in the PLB process (consumer-level, prosumer-level, or hybrid-level for all end-users) and the participation degree (depending on the absorbed/injected current, equal or different to 0 , the 1-P end-user is or not considered in the PLB process). The 3-P prosumers and consumers were treated as the non-switchable end-users.

Testing the methodology has been done using an LV AEDN belonging to a Romanian DNO with 114 end-users integrated into the Smart Metering System during the day when the peak load has been recorded. The performance associated with the time computing in the case of a full participation degree from the end-users (hybrid-level PLB) was better than that obtained in reference [15] (1.13 versus 1.26 seconds), where a similar heuristic PLB algorithm has been used and ran on an EDN having a similar topology. The UF at the level of the CCP has been improved, from 1.30 (unbalanced case, without PLB) to 1.00004. Also, the results have been compared with other possible PLB cases (the consumer-level PLB and prosumer-level PLB), obtaining similar values of the UF (1.028 vs. 1.027), slightly higher than in the hybrid-level PLB. However, all PLB cases lead to an unbalance degree below 1.1 (the target imposed by the DNO). Evaluation of the technical benefits highlighted the significant energy savings between $44.7 \%$ (the case of the prosumer-level PLB) and 58.73\% (the case of the hybrid-level PLB). Also, the phase voltage unbalance rate decreased significantly, from the maximum value of $1.91 \%$ to $0.17 \%$ (the case of the hybrid-level PLB), $0.42 \%$ (the case of the consumer-level PLB), and $0.51 \%$ (the case of the prosumer-level PLB), at the level of the final pole. All results emphasized the effectiveness of the proposed control methodology.

However, their implementation in the AEDNs could be limited by the data communication infrastructure (it must have a high-speed), the master controller from the CCP (having the functions associated with high data acquisition and processing speed), and the 3-P branching for each 1-P end-user.

The authors work now on the extension of the methodology, which considers the storage (or load flexibility) in the PLB process depending on various control configurations. The main objective is to include as many advanced technologies as possible to participate in the PLB process.

Supplementary Materials: The following are available online at http://www.mdpi.com/xxx/s1

Author Contributions: Conceptualization, G.G.; methodology, G.G., L.N. and F.S.; software, G.G.; validation, L.N., E.C., B.C.N, S.F., O.I., and MG.; formal analysis, L.N., E.C., O.I., M.G.; investigation, G.G., B.C.N., F.S., L.N., E.C., M.G., and O.I.; writing-original draft preparation, G.G., B.C.N., F.S., L.N., E.C., M.G. and O.I.; writing-review and editing, G.G., O.I., M.G., and B.C.N.; supervision, G.G., M.G. All authors discussed the results and have agreed with the structure of the paper. All authors have read and agreed to the published version of the manuscript.

Funding: This work was supported by a publications grant of the "Gheorghe Asachi" Technical University of Iasi, project number GI /P19 /2021.

Conflicts of Interest: The authors declare no conflict of interest 


\section{References}

1. Baker, P., Challenges facing distribution system operators in a decarbonised power system. Regulatory Assistance Project, June 2020, Available online: https://www.raponline.org/wp-content/uploads/2020/05/rap-baker-dso-challenges-june-2020final.pdf (accessed on 7 September 2021).

2. Liu, J.; Gao, H.; Ma, Z.; Li,Y, Review and prospect of active distribution system planning. J. Mod. Power Syst. Clean Energy 3 , 457-467 (2015). https://doi.org/10.1007/s40565-015-0170-7

3. European Commission, Clean energy for all Europeans package, 2019, Available online: https://ec.europa.eu/energy/topics/energy-strategy/clean-energy-all-europeans_en (accessed on 7 September 2021).

4. Internal Energy Agency, Digitalization \&Energy, October 2017, Available online: https://iea.blob.core.windows.net/assets/b1e6600c-4e40-4d9c-809d-1d1724c763d5/DigitalizationandEnergy3.pdf (accessed on 7 September 2021).

5. Li, R.; Wang, W.; Chen, Z., Jiang, J., Zhang, W, A Review of Optimal Planning Active Distribution System: Models, Methods, and Future Researches, Energies, 2017, 10, 1715; doi:10.3390/en10111715.

6. Mishra, D.K., \& Ghadi, M.J.,Azizivahed, A., Li, l., Zhang, J., A review on resilience studies in active distribution systems, Renewable and Sustainable Energy Reviews, 2021, 135, 110201, doi: 10.1016/j.rser.2020.110201

7. Beharrysingh, S. Phase Unbalance on Low-Voltage Electricity Networks and Its Mitigation Using Static Balancers. Ph.D. Thesis, Loughborough University, Loughborough, UK, 2014; Available online: https://dspace.lboro.ac.uk/dspacejspui/handle/2134/16252 (accessed on 7 September 2021).

8. Toader, C.; Porumb, R.; Bulac, C.; Tristiu, I. A perspective on current unbalance in low voltage distribution network. In Proceedings of the 9th International Symposium on Advanced Topics in Electrical Engineering (ATEE), Bucharest, Romania, 7-9 May 2015; pp. 741-746.

9. Ojo, A.; Awodele, K.; Sebitosi, A., Load Compensation in a Three-Phase Four Wire Distribution System Considering Unbalance, Neutral Current Elimination and Power Factor Improvement, 2019 Southern African Universities Power Engineering Conference/Robotics and Mechatronics/Pattern Recognition Association of South Africa (SAUPEC/RobMech/PRASA), 2019, pp. 389-394, doi: 10.1109/RoboMech.2019.8704821.

10. Qudah, M., Elhaija, W.A., Widyan, M., Performance analysis of different series compensation schemes of SMIB power system incorporating PV generator, International Transactions on Electrical Energy Systems, 10.1002/2050-7038.12699, 31, 1, (2020).

11. Gebru, Y., Bitew, D., Aberie, H., Gizaw, K., Khan, K., Performance enhancement of radial distribution system using simultaneous network reconfiguration and switched capacitor bank placement, Cogent Engineering, 8:1, DOI: 10.1080/23311916.2021.1897929. 2021.

12. Vai, V.; Suk, S.; Lorm, R.; Chhlonh, C.; Eng, S.; Bun, L. Optimal Reconfiguration in Distribution Systems with Distributed Generations Based on Modified Sequential Switch Opening and Exchange. Appl. Sci. 2021, 11, 2146. https://doi.org/10.3390/app11052146.

13. Shahnia, F.; Wolfs, P.J.; Ghosh, A. Voltage Unbalance Reduction in Low Voltage Feeders by Dynamic Switching of Residential Customers among Three Phases. IEEE Trans. Smart Grid. 2014, 5, 1318-1327.

14. Bao, G.; Ke, S. Load Transfer Device for Solving a Three-Phase Unbalance Problem Under a Low-Voltage Distribution Network. Energies 2019, 12, 2842.

15. Grigoras, G.; Neagu, B.-C.; Gavrilas, M.; Tristiu, I.; Bulac, C. Optimal Phase Load Balancing in Low Voltage Distribution Networks Using a Smart Meter Data-Based Algorithm. Mathematics 2020, 8, 549. https://doi.org/10.3390/math8040549.

16. Liu, B., Meng, K., Dong, Z., Wong, P.K.C., Li, X., Load Balancing in Low-voltage Distribution Network via Phase Reconfiguration: An Efficient Sensitivity-based Approach. IEEE Trans. Power Delivery, 2020, doi:10.1109/TPWRD.2020.3022061.

17. Haq, S.U.; Arif, B.; Khan, A.; Ahmed, J. Automatic Three Phase Load Balancing System by Using Fast Switching Relay in Three Phase Distribution System, International Conference on Power, Energy and Smart Grid, Mirpur, Pakistan, 2018, pp. 1-6.

18. Bao, G.; Ke, S. Load Transfer Device for Solving a Three-Phase Unbalance Problem under a Low-Voltage Distribution Network. Energies vol. 12, 2019, 2842.

19. Homaee, O.; Najafi, A.; Dehghanian, M.; Attar, M.; Falaghi, H. A Practical Approach for Distribution Network Load Balancing by Optimal Re-Phasing of Single-Phase Customers Using Discrete Genetic Algorithm. Int. Trans. Electr. Energy Syst. 2019, 29, e2834.

20. Grigoras, G.; Neagu B.; Scarlatache, F.; Noroc, L; Chelaru, E.; Bi-Level Phase Load Balancing Methodology with ClusteringBased Consumers' Selection Criterion for Switching Device Placement in Low Voltage Distribution Networks, Mathematics, vol. 9, nr. 5, 2021, 542.

21. Sureshkumar, R.; Maithili, P., Three Phase Load Balancing And Energy Loss Reduction In Distribution Network Using Labiew, International Journal of Pure and Applied Mathematics, vol. 116, no. 11, 2017, pp. 181-189.

22. Abril, I.P., Multi-objective optimization of the balancing of phases in primary distribution circuits, International Journal of Electrical Power \& Energy Systems, vol. 82, 2016, pp. 420 - 428.

23. Singh, D.; Misra, RK.; Mishra, S., Distribution system feeder re-phasing considering voltage-dependency of loads, Int. Journal of Electrical Power \& Energy Systems, vol. 76, 2016, pp. 107-119.

24. Siti, W.M.; Jimoh, A.; Nicolae, D., Distribution network phase load balancing as a combinatorial optimization problem using fuzzy logic and Newton-Raphson, Electric Power Systems Research, vol. 81, no. 5, 2011, pp. 1079-1087. 
25. Pan, J.; Liu, J.; Chen, X.; Zhong, K., Three-phase unbalanced load control based on load electricity transfer index, Energy Reports, vol. 7, no. 1, 2021, pp. 312-318.

26. Caldognetto, T.; Tenti, P.; Paolo Mattavelli; Buso, S.; Brandao, D., Cooperative Compensation of Unwanted Current Terms in Low-Voltage Microgrids by Distributed Power-Based Control, 13th Brazilian Power Electronics Conference, Fortaleza, Brazil, 2015.

27. Brandao, D.; Caldognetto, T.; Marafão, F.; Simões, M.; Pomilio, J.; Tenti, P., Centralized Control of Distributed Single-Phase Inverters Arbitrarily Connected to Three-Phase Four-Wire Microgrids, IEEE Transactions on Smart Grid, vol. 8, no. 1, 2017, pp. 437 - 446.

28. Blaud, P.C.; Haurant, P.; Claveau, F.; Lacarrière B.; Chevrel, P.; Mouraud A. , Modelling and control of multi-energy systems through Multi-Prosumer Node and Economic Model Predictive Control, International Journal of Electrical Power \& Energy Systems, vol. 118, 2020, 105778.

29. Mieński, R.; Urbanek, P.; Wasiak, I., Using Energy Storage Inverters of Prosumer Installations for Voltage Control in LowVoltage Distribution Networks. Energies, vol. 14, no. 4, 2021, 1121.

30. Soltani, S.H.; Rashidinejad, M.; Abdollahi, A., Dynamic phase balancing in the smart distribution networks, International Journal of Electrical Power \& Energy Systems, vol. 93, 2017, pp. 374-383.

31. Wang, W.; Yu, N., Phase Balancing in Power Distribution Network with Data Center, ACM SIGMETRICS Performance Evaluation Review, vol. 45, no. 2, 2017 pp. 64-69.

32. Chen, C.; Tsai, C.; Lin, C.; Hsieh, W.; Ku, T., Loading Balance of Distribution Feeders With Loop Power Controllers Considering Photovoltaic Generation," in IEEE Transactions on Power Systems, vol. 26, no. 3, 2011, pp. 1762-1768.

33. Charalambous, A.; Hadjidemetriou, L.; Zacharia, L.; Bintoudi, A.D.; Tsolakis, A.C.; Tzovaras, D.; Kyriakides, E. Phase Balancing and Reactive Power Support Services for Microgrids. Appl. Sci. 2019, 9, 5067. https://doi.org/10.3390/app9235067.

34. Ciontea, C.I.; Iov, F.A., Study of Load Imbalance Influence on Power Quality Assessment for Distribution Networks. Electricity, vol. 2, 2021, pp. 77-90.

35. Kwaśny, L.; Zieliński, D., Control of a Four-wire Hybrid Prosumer Converter for Balancing Utility Grids, Power Electronics and Drives, vol. 6, no. 41, 2021.

36. Drabek, T.; Dybowski, P., Control of prosumer voltage sources in distribution grids, 2020 12th International Conference and Exhibition on Electrical Power Quality and Utilisation, 2020, pp. 1-6.

37. Volosciuc, S.D.; Dragosin, M.E., Prosumer's impact on low voltage distribution networks, 9th International Conference on Manufacturing Science and Education - MSE 2019 "Trends in New Industrial Revolution, MATEC Web Conf., vol. $290,2019$.

38. Grigoras, G.; Neagu, B.-C.; Smart Meter Data-Based Three-Stage Algorithm to Calculate Power and Energy Losses in Low Voltage Distribution Networks. Energies 2019, 12, 3008.

39. Munakata, T.; Barták, R., Logic Programming for Combinatorial Problems, Artificial Intelligence Review, vol. 33, no. 1 -2, pp. 135 - 150, 2010.

40. P. Pillay; M. Manyage, Definitions of Voltage Unbalance, IEEE Power Engineering Review, May 2001, pp. 50-51.

41. M. Bollen, Definitions of voltage unbalance, IEEE Power Engineering Review, vol. 22, no. 11, pp. 49-50, 2002. 